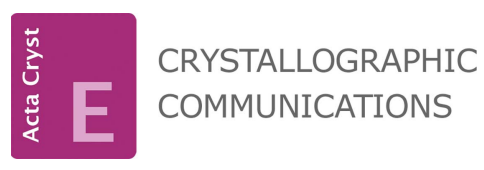

ISSN 2056-9890

Received 18 August 2016

Accepted 29 September 2016

Edited by M. Gdaniec, Adam Mickiewicz

University, Poland

Keywords: crystal structure; copper pyrazolate; trinuclear complex; nanojar; halogen bonding.

CCDC reference: 1507382

Supporting information: this article has supporting information at journals.iucr.org/e

\section{Halogen-bonded network of trinuclear copper(II) 4-iodopyrazolate complexes formed by mutual breakdown of chloroform and nanojars}

\author{
Stuart A. Surmann and Gellert Mezei*
}

Department of Chemistry, Western Michigan University, Kalamazoo, MI 49006. *Correspondence e-mail: gellert.mezei@wmich.edu

Crystals of bis(tetrabutylammonium) di- $\mu_{3}$-chlorido-tris $\left(\mu_{2}\right.$-4-iodopyrazolato$\left.\kappa^{2} N: N^{\prime}\right)$ tris [chloridocuprate(II)] 1,4-dioxane hemisolvate, $\left(\mathrm{C}_{16} \mathrm{H}_{36} \mathrm{~N}\right)_{2}\left[\mathrm{Cu}_{3}\left(\mathrm{C}_{3} \mathrm{H}_{2}\right.\right.$ $\left.\left.\mathrm{IN}_{2}\right)_{3} \mathrm{Cl}_{5}\right] \cdot 0.5 \mathrm{C}_{4} \mathrm{H}_{8} \mathrm{O}$ or $\left(\mathrm{Bu}_{4} \mathrm{~N}\right)_{2}\left[\mathrm{Cu}_{3}^{\mathrm{II}}\left(\mu_{3}-\mathrm{Cl}\right)_{2}(\mu-4-\mathrm{I}-\mathrm{pz})_{3} \mathrm{Cl}_{3}\right] \cdot 0.5 \mathrm{C}_{4} \mathrm{H}_{8} \mathrm{O}$, were obtained by evaporating a solution of $\left(\mathrm{Bu}_{4} \mathrm{~N}\right)_{2}\left[\left\{\mathrm{Cu}^{\mathrm{II}}(\mu-\mathrm{OH})(\mu-4-\mathrm{I}-\mathrm{pz})\right\}_{n} \mathrm{CO}_{3}\right]$ $(n=27-31)$ nanojars in chloroform/1,4-dioxane. The decomposition of chloroform in the presence of oxygen and moisture provides $\mathrm{HCl}$, which leads to the breakdown of nanojars to the title trinuclear copper(II) pyrazolate complex, and possibly $\mathrm{Cu}^{\mathrm{II}}$ ions and free 4 -iodopyrazole. $\mathrm{Cu}^{\mathrm{II}}$ ions, in turn, act as catalyst for the accelerated decomposition of chloroform, ultimately leading to the complete breakdown of nanojars. The crystal structure presented here provides the first structural description of a trinuclear copper(II) pyrazolate complex with iodinesubstituted pyrazoles. In contrast to related trinuclear complexes based on differently substituted 4- $R$-pyrazoles $(R=\mathrm{H}, \mathrm{Cl}, \mathrm{Br}, \mathrm{Me})$, the $\left[\mathrm{Cu}_{3}(\mu-4-\mathrm{I}-\mathrm{pz})_{3} \mathrm{Cl}_{3}\right]$ core in the title complex is nearly planar. This difference is likely a result of the presence of the iodine substituent, which provides a unique, novel feature in copper pyrazolate chemistry. Thus, the iodine atoms form halogen bonds with the terminal chlorido ligands of the surrounding complexes [mean length of $\mathrm{I} \cdots \mathrm{Cl}$ contacts = 3.48 (1) $\AA$ ] , leading to an extended two-dimensional, halogen-bonded network along (110). The cavities within this framework are filled by centrosymmetric 1,4-dioxane solvent molecules, which create further bridges via $\mathrm{C}-\mathrm{H} \cdots \mathrm{Cl}$ hydrogen bonds with terminal chlorido ligands of the trinuclear complex not involved in halogen bonding.

\section{Chemical context}

Nanojars, supramolecular coordination complexes of the formula $\left[\{\mathrm{Cu}(\mu-\mathrm{OH})(\mu-\mathrm{pz})\}_{n}\right.$ anion $]$ (pz = pyrazolate anion; $n$ $=27-36)$, have emerged as a new class of anion encapsulation agents of unparalleled efficiency, which allow the extraction of anions with large hydration energies, such as phosphate, carbonate and sulfate, from water into organic solvents (Mezei, Baran et al., 2004; Fernando et al., 2012; Mezei, 2015; Ahmed, Szymczyna et al., 2016; Ahmed, Calco \& Mezei, 2016; Ahmed \& Mezei, 2016; Ahmed, Hartman \& Mezei, 2016). Trinuclear copper pyrazolate complexes have been identified as key intermediates in the self-assembly mechanism of nanojars from copper(II) nitrate, pyrazole and $\mathrm{NaOH}$ (1:1:2 molar ratio) in the presence of carbonate (Ahmed \& Mezei, 2016). The trinuclear intermediate can be isolated if the amount of available base is reduced (copper:pyrazole:base molar ratio 3:3:4), and can subsequently be converted to nanojars by adding an additional amount of base to reach a 1:1:2 molar ratio. Moreover, nanojars can be broken down to 
the trinuclear complex by acids, which easily protonate the $\mathrm{OH}$ groups of the nanojar. As a consequence, nanojars and the trinuclear pyrazolate complex are in a $\mathrm{pH}$-dependent equilibrium. The sensitivity of nanojars to even very weak acids is further demonstrated by the fact that a weak base, such as $\mathrm{Et}_{3} \mathrm{~N}$, is unable to convert the trinuclear complex to nanojars in solution (e.g., DMF, THF), despite its ability to provide the hydroxide ions needed by the nanojar, in the presence of moisture $\left(\mathrm{Et}_{3} \mathrm{~N}+\mathrm{H}_{2} \mathrm{O} \rightleftharpoons \mathrm{Et}_{3} \mathrm{NH}^{+}+\mathrm{HO}^{-}\right)$. This is due to the acidity of the conjugate acid, the triethylammonium cation $\left(\mathrm{p} K_{\mathrm{a}}=10.75\right.$ in $\mathrm{H}_{2} \mathrm{O}$ ), which would form in the process (Mezei, 2016). Nevertheless, nanojars can be obtained using $\mathrm{Et}_{3} \mathrm{~N}$ if the solution is diluted with excess water, which leads to the precipitation of hydrophobic nanojars (Fernando et al., 2012).

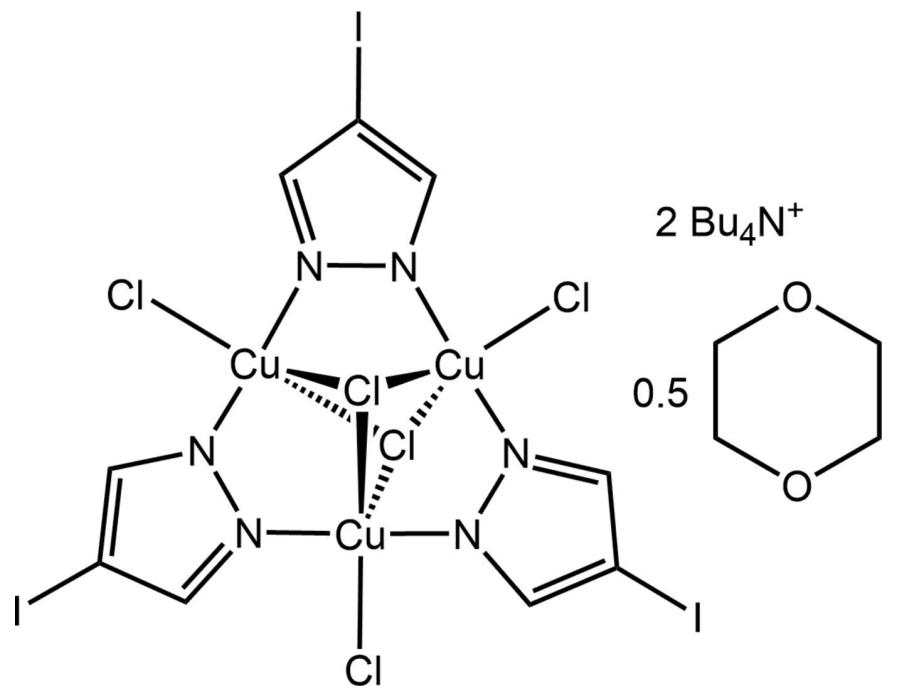

New evidence supporting the vulnerability of nanojars to acids emerges from an unexpected source. An attempt to grow single crystals from a solution of $\left(\mathrm{Bu}_{4} \mathrm{~N}\right)_{2}[\{\mathrm{Cu}(\mu-\mathrm{OH})(\mu-4-\mathrm{I}-$ pz) $\left.\}_{n} \mathrm{CO}_{3}\right](n=27-31)$ (Ahmed, Calco et al., 2016) in chloroform/1,4-dioxane provided, instead of the expected nanojars, crystals of $\left(\mathrm{Bu}_{4} \mathrm{~N}\right)_{2}\left[\mathrm{Cu}_{3}\left(\mu_{3}-\mathrm{Cl}\right)_{2}(\mu-4-\mathrm{I}-\mathrm{pz})_{3} \mathrm{Cl}_{3}\right] \cdot 0.5$ dioxane (Mezei \& Raptis, 2004), accompanied by a color change of the solution from blue to green. The chloride ions originating from $\mathrm{CHCl}_{3}$ is not surprising, as chloroform has long been known to slowly decompose in the presence of air and moisture producing $\mathrm{HCl}$ and phosgene $\left(\mathrm{CHCl}_{3}+\frac{1}{2} \mathrm{O}_{2} \rightarrow \mathrm{COCl}_{2}+\mathrm{HCl}\right)$ (Baskerville \& Hamor, 1912). The latter can hydrolyze to provide further amounts of $\mathrm{HCl}$, and $\mathrm{CO}_{2}\left(\mathrm{COCl}_{2}+\mathrm{H}_{2} \mathrm{O} \rightarrow\right.$ $\left.2 \mathrm{HCl}+\mathrm{CO}_{2}\right)$. What is surprising though is the large amount of chloride formed in a relatively short period of time ( $c a 48$ chloride ions per nanojar). Chloroform preserved with ethanol $(0.5-1 \%)$, such as the one used here for crystal growing, is much more stable than the pure form and it does not decompose at a significant rate. This points to a decomposition catalyzed by the dissolved nanojars, possibly aided by light. A search of the literature shows that various classes of compounds have been found to catalyze the photodecomposition of chloroform (Semeluk \& Unger, 1963; Peña \& Hoggard, 2010; Muñoz et al., 2008; Peña et al., 2014; Peña et al., 2009), including simple copper(II) complexes (Harvey \& Hoggard, 2014). A balanced equation of the reaction between nanojars of different sizes and $\mathrm{HCl}$, producing the title trinuclear complex, is given below:

$3\left[\left\{\mathrm{Cu}(\mu-\mathrm{OH})(\mu-4-\mathrm{Ipz})_{n} \mathrm{CO}_{3}\right]^{2-}+5 n \mathrm{HCl} \rightarrow n\left[\mathrm{Cu}_{3}\left(\mu_{3}-\mathrm{Cl}\right)_{2^{-}}\right.\right.$ $\left.(\mu-4-\mathrm{Ipz})_{3} \mathrm{Cl}_{3}\right]^{2-}+(2 n-6) \mathrm{H}_{3} \mathrm{O}^{+}+(n+9) \mathrm{H}_{2} \mathrm{O}+3 \mathrm{CO}_{2}(n=$ 27-31).

\section{Structural commentary}

The title compound contains a nearly planar $\mathrm{Cu}_{3}(\mu-4-\mathrm{I}-\mathrm{pz})_{3}$ core (Fig. 1): the best-fit planes of the three 4-iodopyrazolate units form dihedral angles of $2.1(2), 2.0(1)$ and $6.5(1)^{\circ}$, respectively, with the $\mathrm{Cu}_{3}$-plane. Each $\mathrm{Cu}$ atom has a distorted trigonal-bipyramidal coordination geometry and is bound to a terminal $\mathrm{Cl}$ atom (one $\mathrm{Cl}$ atom disordered over two positions, $60 / 40$ occupancy) at an average $\mathrm{Cu}-\mathrm{Cl}$ distance of 2.32 (3) $\AA$. The $\mathrm{Cu}_{3}$ unit is additionally capped by two $\mathrm{Cl}$ atoms, one on each side of the complex, at distances of 1.683 (1) and 1.799 (1) $\AA$ from the $\mathrm{Cu}_{3}$-plane, respectively [average $\mathrm{Cu}-\mathrm{Cl}$ distances $=2.58$ (7) and 2.66 (9) $\AA$ ]. The two capping $\mathrm{Cl}$ atoms impart an overall 2-charge to the complex, which is balanced by two tetrabutylammonium counter-ions. Other bond lengths and angles within the $\mathrm{Cu}_{3}\left(\mu_{3}-\mathrm{Cl}\right)_{2}(\mu-4-\mathrm{I}-\mathrm{pz})_{3} \mathrm{Cl}_{3}$ complex are similar to the ones found in related complexes (Angaridis et al., 2002; Mezei \& Raptis, 2004; Mezei et al., 2006): $\mathrm{Cu}-\mathrm{N}$ bond lengths average $1.936(10) \AA, \mathrm{N}-\mathrm{Cu}-\mathrm{N}$ angles average $173(3)^{\circ}, \mathrm{Cl}-\mathrm{Cu}-\mathrm{Cl}$ angles average $125(9)$ and $152(9)^{\circ}$, respectively, and intramolecular $\mathrm{Cu} \cdots \mathrm{Cu}$ distances are 3.378 (1), 3.419 (1) and 3.390 (1) А̊.

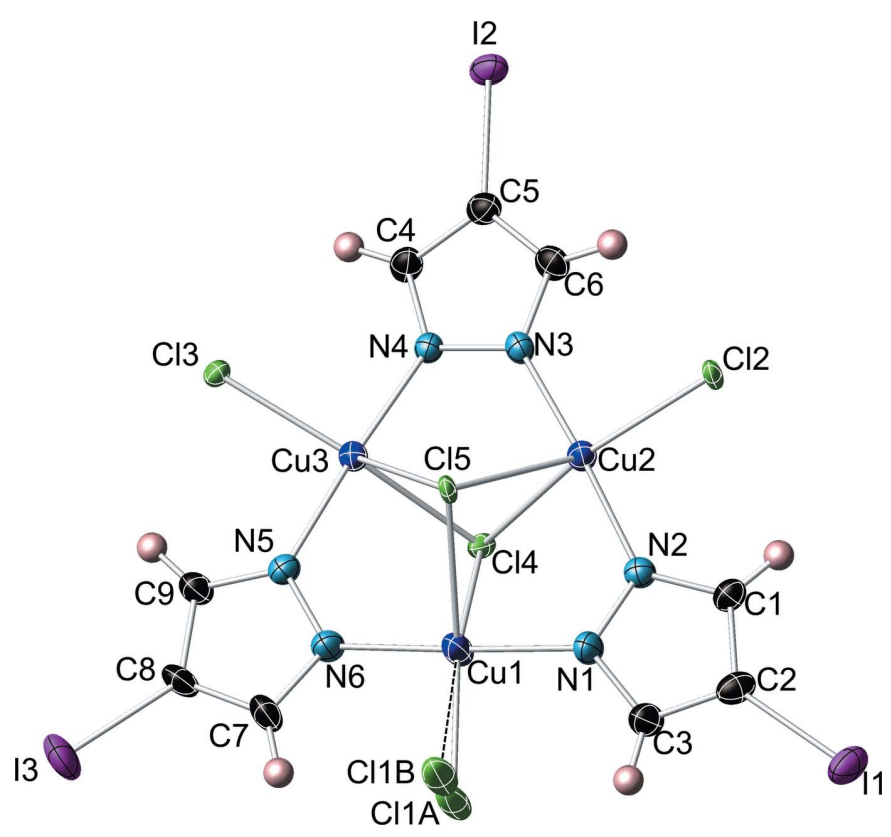

Figure 1

Displacement ellipsoid plot (50\% probability level) of the title trinuclear copper pyrazolate complex anion, showing the atom-labeling scheme (counter-ions and solvent molecule omitted). 


\section{Supramolecular features}

The intermolecular distances between iodine substituents of the pyrazole units and the terminal chlorine atoms of adjacent complexes are less than the sum of the van der Waals radii (Bondi, 1964) of iodine and chlorine atoms (3.73 A). Thus, a halogen-bonded (Cavallo et al., 2016; Gilday et al., 2015) sheet based on $\mathrm{C}-\mathrm{I} \cdots \mathrm{Cl}-\mathrm{Cu}$ interactions (Fig. 2) is generated parallel to the (110) plane (and $c$ axis); $\mathrm{I} \cdots \mathrm{Cl}$ distances and C$\mathrm{I} \cdot \mathrm{Cl}$ angles are shown in Table 1 . Bifurcated halogen bonds are noted between $\mathrm{Cl} 1 A / \mathrm{Cl} 1 B$ and $\mathrm{I}^{\prime}{ }^{\prime}$ and $\mathrm{I}^{\prime}{ }^{\prime}$. The formation of the extended halogen-bonded network might account for the near-planarity of the title complex, as opposed to related complexes with unsubstituted or differently substituted 4- $R$ pyrazoles $(R=\mathrm{H}, \mathrm{Cl}, \mathrm{Br}, \mathrm{Me}$; Angaridis et al., 2002; Mezei \& Raptis, 2004), which do not form intermolecular halogen bonds and are severely distorted from planarity. Additionally, the dioxane solvent molecule, which is located around an inversion center, forms $\mathrm{C}-\mathrm{H} \cdots \mathrm{Cl}$ hydrogen bonds with terminal chlorido ligands of the trinuclear complex [C43 ‥Cl2: 3.751 (10); H43B ‥Cl2: 2.83; C43-H43B: $0.97 \AA$; $\mathrm{C} 43-\mathrm{H} 43 \mathrm{~b} \cdots \mathrm{Cl} 2$ : $\left.160(5)^{\circ}\right]$, creating further bridges within the two-dimensional framework.
Table 1

Halogen-bond geometry $\left(\AA,^{\circ}\right)$.

\begin{tabular}{lll}
\hline$D-X \cdots Y$ & $X \cdots Y$ & $D-X \cdots Y$ \\
\hline $\mathrm{C} 2-\mathrm{I} 1 \cdots \mathrm{Cl} 1 A^{\mathrm{i}}$ & $3.516(4)$ & $152.0(2)$ \\
$\mathrm{C} 2-\mathrm{I} 1 \cdots \mathrm{Cl} 1 B^{\mathrm{i}}$ & $3.362(5)$ & $164.3(2)$ \\
$\mathrm{C} 5-\mathrm{I} 2 \cdots \mathrm{Cl}{ }^{\text {iii }}$ & $3.569(1)$ & $165.2(2)$ \\
$\mathrm{C} 8-\mathrm{I} 3 \cdots \mathrm{Cl} 1 A^{\mathrm{iv}}$ & $3.438(4)$ & $154.4(2)$ \\
$\mathrm{C} 8-\mathrm{I} 3 \cdots \mathrm{Cl} 1 B^{\mathrm{iv}}$ & $3.486(5)$ & $154.2(2)$ \\
\hline
\end{tabular}

Symmetry codes: (i) $-x+2,-y,-z$; (ii) $-x+1,-y-1,-z+1$; (iii) $-x+1,-y-1$, $-z+1 ;$ (iv) $-x+1,-y-1,-z$.

\section{Database survey}

A search of the Cambridge Structural Database (Groom et al., 2016) reveals only seven metal complexes that contain a 4-iodopyrazole moiety, either in its neutral, monodentate form (Guzei \& Winter, 1997; Govor et al., 2012; Song et al., 2013; da Silva et al., 2015), or in its deprotonated, bidentate form (Heeg et al., 2010; Song et al., 2013). Of these, only one is a $\mathrm{Cu}^{\mathrm{II}}$ complex (Song et al., 2013). Hence, the crystal structure presented here offers the first solid-state structural description

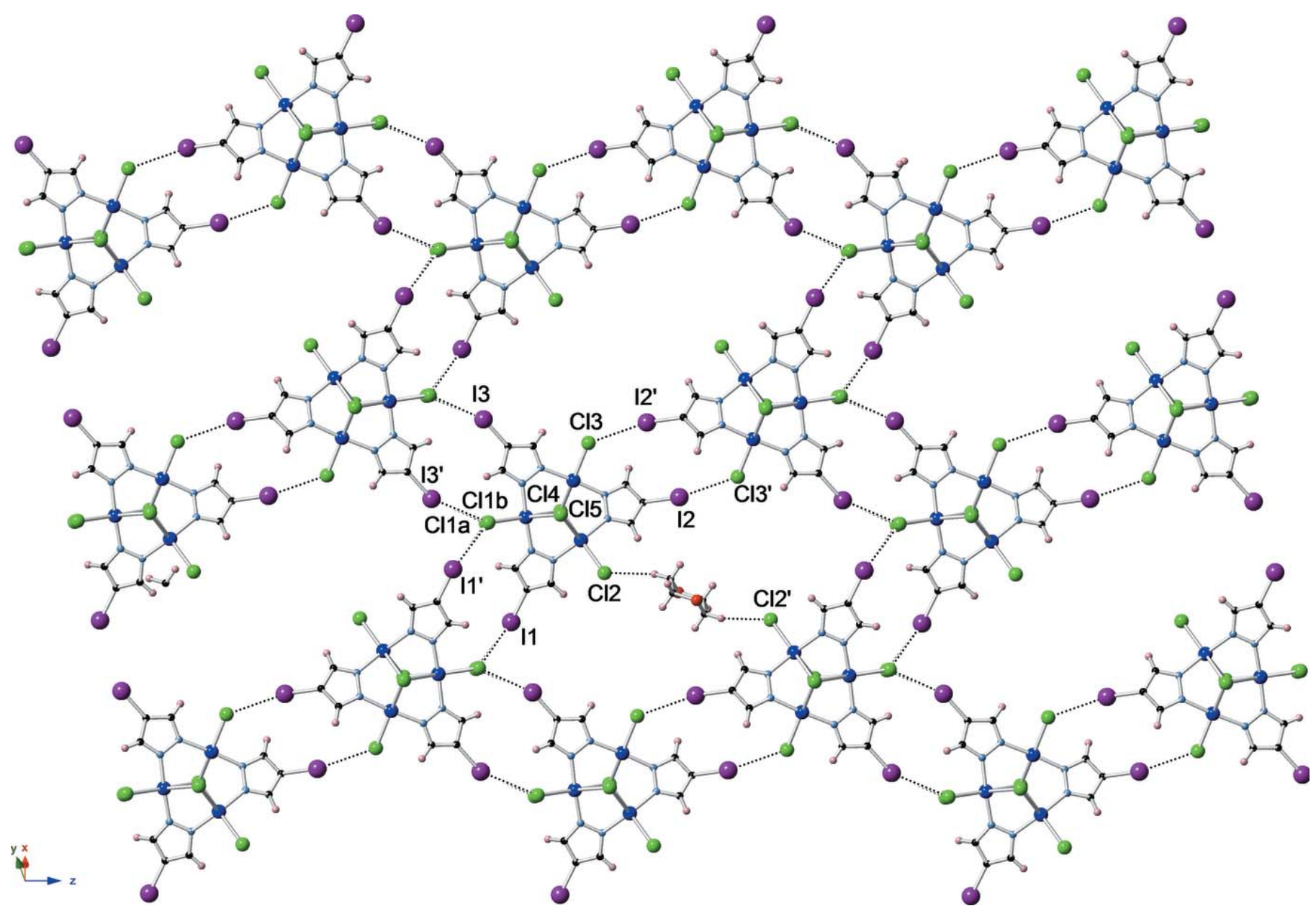

Figure 2

Two-dimensional sheet [along (110)] formed by intermolecular iodine-chlorine halogen bonding (only one dioxane solvent molecule and no counterions are shown). Halogen bonds and $\mathrm{C}-\mathrm{H} \cdots \mathrm{Cl}$ hydrogen bonds are indicated by dotted lines. 
Table 2

Experimental details.

\begin{tabular}{|c|c|}
\hline \multicolumn{2}{|l|}{ Crystal data } \\
\hline Chemical formula & $\begin{array}{l}\left(\mathrm{C}_{16} \mathrm{H}_{36} \mathrm{~N}\right)_{2}\left[\mathrm{Cu}_{3}\left(\mathrm{C}_{3} \mathrm{H}_{2} \mathrm{IN}_{2}\right)_{3^{-}}\right. \\
\left.\mathrm{Cl}_{5}\right] \cdot 0.5 \mathrm{C}_{4} \mathrm{H}_{8} \mathrm{O}\end{array}$ \\
\hline$M_{\mathrm{r}}$ & 1475.73 \\
\hline Crystal system, space group & Triclinic, $P \overline{1}$ \\
\hline Temperature $(\mathrm{K})$ & 100 \\
\hline$a, b, c(\AA)$ & $\begin{array}{l}11.3604(2), 11.5688(2) \\
23.2200(3)\end{array}$ \\
\hline$\alpha, \beta, \gamma\left(^{\circ}\right)$ & 103.707 (1), 90.409 (1), 93.654 (1) \\
\hline$V\left(\AA^{3}\right)$ & $2958.00(8)$ \\
\hline$Z$ & 2 \\
\hline Radiation type & Мо $K \alpha$ \\
\hline$\mu\left(\mathrm{mm}^{-1}\right)$ & 2.90 \\
\hline Crystal size $(\mathrm{mm})$ & $0.65 \times 0.43 \times 0.03$ \\
\hline \multicolumn{2}{|l|}{ Data collection } \\
\hline Diffractometer & Bruker APEXII CCD \\
\hline Absorption correction & $\begin{array}{l}\text { Multi-scan (SADABS; Bruker, } \\
\text { 2014) }\end{array}$ \\
\hline$T_{\min }, T_{\max }$ & $0.486,0.746$ \\
\hline $\begin{array}{l}\text { No. of measured, independent and } \\
\text { observed }[I>2 \sigma(I)] \text { reflections }\end{array}$ & $136857,14685,11845$ \\
\hline$R_{\text {int }}$ & 0.056 \\
\hline$(\sin \theta / \lambda)_{\max }\left(\AA^{-1}\right)$ & 0.668 \\
\hline \multicolumn{2}{|l|}{ Refinement } \\
\hline$R\left[F^{2}>2 \sigma\left(F^{2}\right)\right], w R\left(F^{2}\right), S$ & $0.054,0.153,1.02$ \\
\hline No. of reflections & 14685 \\
\hline No. of parameters & 642 \\
\hline No. of restraints & 12 \\
\hline $\mathrm{H}$-atom treatment & H-atom parameters constrained \\
\hline$\Delta \rho_{\max }, \Delta \rho_{\min }\left(\mathrm{e} \AA^{-3}\right)$ & $3.54,-2.89$ \\
\hline
\end{tabular}

Computer programs: APEX2 and SAINT (Bruker, 2014), SHELXS97 (Sheldrick, 2008), SHELXL2014/6 (Sheldrick, 2015) and CrystalMaker (Palmer, 2014).

of a trinuclear copper(II) pyrazolate complex bearing 4-iodopyrazolate ligands.

\section{Synthesis and crystallization}

The synthesis of $\left(\mathrm{Bu}_{4} \mathrm{~N}\right)_{2}\left[\{\mathrm{Cu}(\mu-\mathrm{OH})(\mu-4-\mathrm{I}-\mathrm{pz})\}_{n} \mathrm{CO}_{3}\right](n=$ 27-31) was described earlier (Ahmed Calco \& Mezei, 2016). Green plate-like crystals of the title compound were obtained by slow evaporation of a chloroform/1,4-dioxane (1 mL each) solution of $\left(\mathrm{Bu}_{4} \mathrm{~N}\right)_{2}\left[\{\mathrm{Cu}(\mu-\mathrm{OH})(\mu-4-\mathrm{I}-\mathrm{pz})\}_{n} \mathrm{CO}_{3}\right](20 \mathrm{mg})$.

\section{Refinement}

Crystal data, data collection and structure refinement details are summarized in Table 2. $\mathrm{C}-\mathrm{H}$ hydrogen atoms were placed in idealized positions and refined using a riding model. One of the three terminal $\mathrm{Cl}$-atoms is disordered over two positions (60/40). Two terminal $\mathrm{CH}_{2} \mathrm{CH}_{3}$ groups of one tetrabutylammonium counter-ion and another $\mathrm{CH}_{2} \mathrm{CH}_{3}$ group of the other counter-ion are disordered over two positions (60/40); $\mathrm{C}-\mathrm{H}$ bond-length restraints were used for the disordered $\mathrm{C}$ atoms. Residual electron density of $3.52 \mathrm{e}^{-3}$ is found at $0.83 \AA$ from heavy atom I3, due to Fourier truncation ripples.

\section{Acknowledgements}

This material is based on work supported by the National Science Foundation under Grant No. CHE-1404730.

\section{References}

Ahmed, B. M., Calco, B. \& Mezei, G. (2016). Dalton Trans. 45, 83278339.

Ahmed, B. M., Hartman, C. K. \& Mezei, G. (2016). Inorg. Chem. 55, doi:10.1021/acs.inorgchem.6b01909.

Ahmed, B. M. \& Mezei, G. (2016). Inorg. Chem. 55, 7717-7728.

Ahmed, B. M., Szymczyna, B. R., Jianrattanasawat, S., Surmann, S. A. \& Mezei, G. (2016). Chem. Eur. J. 22, 5499-5503.

Angaridis, P. A., Baran, P., Boča, R., Cervantes-Lee, F., Haase, W., Mezei, G., Raptis, R. G. \& Werner, R. (2002). Inorg. Chem. 41, 2219-2228.

Baskerville, C. \& Hamor, W. A. (1912). J. Ind. Eng. Chem. 4, 278-288.

Bondi, A. (1964). J. Phys. Chem. 68, 441-451.

Bruker (2014). APEX2, SAINT and SADABS. Bruker AXS Inc., Madison, Wisconsin, USA.

Cavallo, G., Metrangolo, P., Milani, R., Pilati, T., Priimagi, A., Resnati, G. \& Terraneo, G. (2016). Chem. Rev. 116, 2478-2601.

Fernando, I. R., Surmann, S. A., Urech, A. A., Poulsen, A. M. \& Mezei, G. (2012). Chem. Commun. 48, 6860-6862.

Gilday, L. C., Robinson, S. W., Barendt, T. A., Langton, M. J., Mullaney, B. R. \& Beer, P. D. (2015). Chem. Rev. 115, 7118-7195.

Govor, E. V., Chakraborty, I., Piñero, D. M., Baran, P., Sanakis, Y. \& Raptis, R. G. (2012). Polyhedron, 45, 55-60.

Groom, C. R., Bruno, I. J., Lightfoot, M. P. \& Ward, S. C. (2016). Acta Cryst. B72, 171-179.

Guzei, I. A. \& Winter, C. H. (1997). Inorg. Chem. 36, 4415-4420.

Harvey, B. M. \& Hoggard, P. E. (2014). Photochem. Photobiol. 90, 1234-1242.

Heeg, M. J., Yu, Z. \& Winter, C. H. (2010). Private Communication (refcode DAGDUM). CCDC, Cambridge, England.

Mezei, G. (2015). Chem. Commun. 51, 10341-10344.

Mezei, G. (2016). Acta Cryst. E72, 1064-1067.

Mezei, G., Baran, P. \& Raptis, R. G. (2004). Angew. Chem. Int. Ed. 43, 574-577.

Mezei, G. \& Raptis, R. G. (2004). Inorg. Chim. Acta, 357, 3279-3288.

Mezei, G., Raptis, R. G. \& Telser, J. (2006). Inorg. Chem. 45, 88418843.

Muñoz, Z., Cohen, A. S., Nguyen, L. M., McIntosh, T. A. \& Hoggard, P. E. (2008). Photochem. Photobiol. Sci. 7, 337-343.

Palmer, D. C. (2014). CrystalMaker. CrystalMaker Software Ltd, Begbroke, England.

Peña, L. A., Chan, A. M., Cohen, L. R., Hou, K., Harvey, B. M. \& Hoggard, P. E. (2014). Photochem. Photobiol. 90, 760-766.

Peña, L. A. \& Hoggard, P. E. (2010). J. Mol. Catal. A Chem. 327, 20 24.

Peña, L. A., Seidl, A. J., Cohen, L. R. \& Hoggard, P. E. (2009). Transition Met. Chem. 34, 135-141.

Semeluk, G. P. \& Unger, I. (1963). Nature, 198, 853-855.

Sheldrick, G. M. (2008). Acta Cryst. A64, 112-122.

Sheldrick, G. M. (2015). Acta Cryst. C71, 3-8.

Silva, C. da, Ribeiro, L. B., Furuno, C. C., da Cunha, G. A., de Souza, R. F. F., Netto, A. V. G., Mauro, A. E., Frem, R. C. G., Fernandes, J. A., Almeida Paz, F. A., Marino, L. B., Pavan, F. R. \& Leite, C. Q. F. (2015). Polyhedron, 100, 10-16.

Song, G., Sun, Q., Hou, Y.-N., Zhan, R., Wei, D.-M., Shi, Z. \& Xing, Y.-H. (2013). Chin. J. Inorg. Chem. 29, 2150-2156. 


\section{supporting information}

Acta Cryst. (2016). E72, 1517-1520 [https://doi.org/10.1107/S205698901601536X]

\section{Halogen-bonded network of trinuclear copper(II) 4-iodopyrazolate complexes formed by mutual breakdown of chloroform and nanojars}

\section{Stuart A. Surmann and Gellert Mezei}

Computing details

Data collection: APEX2 (Bruker, 2014); cell refinement: SAINT (Bruker, 2014); data reduction: SAINT (Bruker, 2014); program(s) used to solve structure: SHELXS97 (Sheldrick, 2008); program(s) used to refine structure: SHELXL2014/6 (Sheldrick, 2015); molecular graphics: CrystalMaker (Palmer, 2014); software used to prepare material for publication: CrystalMaker (Palmer, 2014).

Bis(tetrabutylammonium) di- $\mu_{3}$-chlorido-tris( $\mu$-4-iodopyrazolato- $\left.\kappa^{2} N: N^{\prime}\right)$ tris[chloridocuprate(II)] 1,4-dioxane hemisolvate

Crystal data

$\left(\mathrm{C}_{16} \mathrm{H}_{36} \mathrm{~N}\right)_{2}\left[\mathrm{Cu}_{3} \mathrm{Cl}_{5}\left(\mathrm{C}_{3} \mathrm{H}_{2} \mathrm{IN}_{2}\right)_{3} \mathrm{Cl}_{5}\right] \cdot 0.5 \mathrm{C}_{4} \mathrm{H}_{8} \mathrm{O}$

$M_{r}=1475.73$

Triclinic, $P \overline{1}$

$a=11.3604(2) \AA$

$b=11.5688(2) \AA$

$c=23.2200(3) \AA$

$\alpha=103.707(1)^{\circ}$

$\beta=90.409(1)^{\circ}$

$\gamma=93.654(1)^{\circ}$

$V=2958.00(8) \AA^{3}$

Data collection

Bruker APEXII CCD

diffractometer

$\varphi$ and $\omega$ scans

Absorption correction: multi-scan

(SADABS; Bruker, 2014)

$T_{\min }=0.486, T_{\max }=0.746$

136857 measured reflections

Refinement

Refinement on $F^{2}$

Least-squares matrix: full

$R\left[F^{2}>2 \sigma\left(F^{2}\right)\right]=0.054$

$w R\left(F^{2}\right)=0.153$

$S=1.02$

14685 reflections

642 parameters

12 restraints
$Z=2$

$F(000)=1470$

$D_{\mathrm{x}}=1.657 \mathrm{Mg} \mathrm{m}^{-3}$

Mo $K \alpha$ radiation, $\lambda=0.71073 \AA$

Cell parameters from 9763 reflections

$\theta=2.2-28.1^{\circ}$

$\mu=2.90 \mathrm{~mm}^{-1}$

$T=100 \mathrm{~K}$

Plate, green

$0.65 \times 0.43 \times 0.03 \mathrm{~mm}$

14685 independent reflections

11845 reflections with $I>2 \sigma(I)$

$R_{\text {int }}=0.056$

$\theta_{\max }=28.3^{\circ}, \theta_{\min }=0.9^{\circ}$

$h=-15 \rightarrow 15$

$k=-15 \rightarrow 15$

$l=-30 \rightarrow 30$

Hydrogen site location: inferred from neighbouring sites

$\mathrm{H}$-atom parameters constrained

$w=1 /\left[\sigma^{2}\left(F_{\mathrm{o}}^{2}\right)+(0.0646 P)^{2}+28.784 P\right]$

where $P=\left(F_{\mathrm{o}}^{2}+2 F_{\mathrm{c}}^{2}\right) / 3$

$(\Delta / \sigma)_{\max }=0.001$

$\Delta \rho_{\max }=3.54 \mathrm{e} \AA^{-3}$

$\Delta \rho_{\min }=-2.89$ e $\AA^{-3}$ 


\section{Special details}

Geometry. All esds (except the esd in the dihedral angle between two 1.s. planes) are estimated using the full covariance matrix. The cell esds are taken into account individually in the estimation of esds in distances, angles and torsion angles; correlations between esds in cell parameters are only used when they are defined by crystal symmetry. An approximate (isotropic) treatment of cell esds is used for estimating esds involving l.s. planes.

Fractional atomic coordinates and isotropic or equivalent isotropic displacement parameters $\left(\hat{A}^{2}\right)$

\begin{tabular}{|c|c|c|c|c|c|}
\hline & $x$ & $y$ & $z$ & $U_{\text {iso }} * / U_{\text {eq }}$ & Occ. $(<1)$ \\
\hline I1 & $1.03331(4)$ & $1.16081(4)$ & $0.94854(2)$ & $0.03491(11)$ & \\
\hline $\mathrm{I} 2$ & $0.60033(4)$ & $0.67453(3)$ & $0.48116(2)$ & $0.02938(10)$ & \\
\hline I3 & $0.33478(5)$ & $0.37443(5)$ & $0.92175(2)$ & $0.05065(15)$ & \\
\hline $\mathrm{Cu} 1$ & $0.68348(7)$ & $0.74045(8)$ & $0.86260(3)$ & 0.03445 (19) & \\
\hline $\mathrm{Cu} 2$ & $0.76595(6)$ & $0.82666(6)$ & $0.73883(3)$ & 0.02209 (14) & \\
\hline $\mathrm{Cu} 3$ & $0.54352(6)$ & $0.60997(6)$ & $0.73172(3)$ & 0.01939 (14) & \\
\hline Cl1A & $0.7088(4)$ & $0.7605(4)$ & 0.96245 (14) & $0.0517(10)$ & 0.6 \\
\hline Cl1B & $0.7610(5)$ & $0.7019(5)$ & $0.9490(2)$ & $0.0456(13)$ & 0.4 \\
\hline $\mathrm{Cl} 2$ & $0.91814(10)$ & $0.91245(10)$ & $0.69157(5)$ & $0.0170(2)$ & \\
\hline $\mathrm{Cl} 3$ & $0.43631(10)$ & $0.44226(10)$ & $0.67503(5)$ & 0.0154 (2) & \\
\hline $\mathrm{Cl} 4$ & $0.55813(9)$ & $0.82369(10)$ & $0.78789(5)$ & $0.0150(2)$ & \\
\hline $\mathrm{Cl} 5$ & $0.77907(9)$ & $0.62234(9)$ & $0.76421(4)$ & 0.01119 (19) & \\
\hline $\mathrm{O} 1$ & $0.9089(5)$ & $0.0637(5)$ & $0.5297(2)$ & $0.0456(12)$ & \\
\hline N1 & $0.7933(4)$ & $0.8756(5)$ & $0.8653(2)$ & $0.0274(10)$ & \\
\hline $\mathrm{N} 2$ & 0.8247 (4) & 0.9129 (4) & 0.81648 (19) & $0.0208(9)$ & \\
\hline N3 & $0.6824(4)$ & $0.7512(4)$ & $0.66521(19)$ & $0.0213(9)$ & \\
\hline N4 & 0.5915 (4) & $0.6671(4)$ & $0.66256(19)$ & $0.0207(9)$ & \\
\hline N5 & $0.5023(4)$ & $0.5613(4)$ & $0.80432(19)$ & $0.0223(9)$ & \\
\hline N6 & $0.5610(4)$ & $0.6148(5)$ & $0.8565(2)$ & $0.0269(10)$ & \\
\hline N7 & $0.1835(4)$ & $0.7648(5)$ & $0.8299(3)$ & $0.0336(12)$ & \\
\hline N8 & $0.7491(5)$ & $0.2456(5)$ & $0.6678(3)$ & $0.0338(12)$ & \\
\hline $\mathrm{C} 1$ & $0.9002(5)$ & $1.0111(5)$ & $0.8331(2)$ & $0.0219(10)$ & \\
\hline H1 & 0.9350 & 1.0540 & 0.8078 & $0.026^{*}$ & \\
\hline $\mathrm{C} 2$ & $0.9171(5)$ & $1.0369(5)$ & $0.8945(2)$ & $0.0232(11)$ & \\
\hline $\mathrm{C} 3$ & $0.8487(5)$ & $0.9500(6)$ & $0.9130(3)$ & 0.0295 (13) & \\
\hline H3 & 0.8418 & 0.9436 & 0.9520 & $0.035^{*}$ & \\
\hline $\mathrm{C} 4$ & $0.5500(5)$ & $0.6341(5)$ & $0.6066(2)$ & $0.0227(11)$ & \\
\hline $\mathrm{H} 4$ & 0.4874 & 0.5782 & 0.5929 & $0.027^{*}$ & \\
\hline $\mathrm{C} 5$ & $0.6150(5)$ & $0.6965(5)$ & $0.5720(2)$ & $0.0233(11)$ & \\
\hline C6 & $0.6974(5)$ & $0.7683(5)$ & $0.6107(2)$ & $0.0232(11)$ & \\
\hline H6 & 0.7545 & 0.8206 & 0.6004 & $0.028^{*}$ & \\
\hline $\mathrm{C} 7$ & $0.5171(5)$ & $0.5648(6)$ & $0.8991(3)$ & $0.0294(12)$ & \\
\hline H7 & 0.5424 & 0.5846 & 0.9386 & $0.035^{*}$ & \\
\hline $\mathrm{C} 8$ & $0.4282(6)$ & $0.4787(6)$ & $0.8748(3)$ & $0.0314(13)$ & \\
\hline C9 & $0.4229(5)$ & $0.4791(5)$ & $0.8146(2)$ & $0.0260(11)$ & \\
\hline H9 & 0.3721 & 0.4299 & 0.7863 & $0.031 *$ & \\
\hline $\mathrm{C} 10$ & $0.0713(6)$ & $0.7144(9)$ & $0.8526(4)$ & $0.056(2)$ & \\
\hline $\mathrm{H} 10 \mathrm{~A}$ & 0.0536 & 0.7664 & 0.8905 & $0.067 *$ & \\
\hline $\mathrm{H} 10 \mathrm{~B}$ & 0.0069 & 0.7160 & 0.8252 & $0.067 *$ & \\
\hline
\end{tabular}




\begin{tabular}{|c|c|c|c|c|c|}
\hline C11 & $0.0750(8)$ & $0.5893(13)$ & $0.8606(7)$ & $0.104(5)$ & \\
\hline H11A & 0.1434 & 0.5818 & 0.8844 & $0.125^{*}$ & 0.6 \\
\hline H11B & 0.0769 & 0.5321 & 0.8226 & $0.125^{*}$ & 0.6 \\
\hline $\mathrm{H} 11 \mathrm{C}$ & 0.1322 & 0.5965 & 0.8927 & $0.125^{*}$ & 0.4 \\
\hline H11D & 0.1128 & 0.5470 & 0.8252 & $0.125^{*}$ & 0.4 \\
\hline $\mathrm{C} 12 \mathrm{~A}$ & $-0.0422(11)$ & $0.5702(14)$ & $0.8934(7)$ & $0.047(3)$ & 0.6 \\
\hline $\mathrm{H} 12 \mathrm{~A}$ & -0.1102 & 0.5837 & 0.8710 & $0.056^{*}$ & 0.6 \\
\hline H12B & -0.0415 & 0.6233 & 0.9326 & $0.056^{*}$ & 0.6 \\
\hline $\mathrm{C} 13 \mathrm{~A}$ & $-0.0432(18)$ & $0.4346(17)$ & $0.8972(12)$ & $0.112(10)$ & 0.6 \\
\hline $\mathrm{H} 13 \mathrm{~A}$ & -0.0392 & 0.3843 & 0.8580 & $0.168^{*}$ & 0.6 \\
\hline H13B & -0.1146 & 0.4136 & 0.9153 & $0.168^{*}$ & 0.6 \\
\hline $\mathrm{H} 13 \mathrm{C}$ & 0.0235 & 0.4241 & 0.9206 & $0.168 *$ & 0.6 \\
\hline C12B & -0.020 & $0.500(3)$ & $0.8721(10)$ & $0.077(9)$ & 0.4 \\
\hline $\mathrm{H} 12 \mathrm{C}$ & -0.0074 & 0.4199 & 0.8491 & $0.093 *$ & 0.4 \\
\hline H12D & -0.0977 & 0.5209 & 0.8630 & $0.093 *$ & 0.4 \\
\hline C13B & $-0.002(3)$ & $0.510(3)$ & $0.9386(10)$ & $0.087(9)$ & 0.4 \\
\hline H13D & 0.0684 & 0.4729 & 0.9453 & $0.131 *$ & 0.4 \\
\hline H13E & -0.0687 & 0.4705 & 0.9530 & $0.131 *$ & 0.4 \\
\hline $\mathrm{H} 13 \mathrm{~F}$ & 0.0047 & 0.5924 & 0.9593 & $0.131 *$ & 0.4 \\
\hline C14 & $0.2866(6)$ & $0.7694(7)$ & $0.8727(3)$ & 0.0363 (14) & \\
\hline $\mathrm{H} 14 \mathrm{~A}$ & 0.3572 & 0.7979 & 0.8556 & $0.044 *$ & \\
\hline H14B & 0.2980 & 0.6886 & 0.8758 & $0.044^{*}$ & \\
\hline C15 & $0.2755(7)$ & $0.8467(9)$ & $0.9350(3)$ & $0.056(2)$ & \\
\hline $\mathrm{H} 15 \mathrm{~A}$ & 0.2121 & 0.8136 & 0.9552 & $0.067^{*}$ & 0.6 \\
\hline H15B & 0.2575 & 0.9267 & 0.9332 & $0.067^{*}$ & 0.6 \\
\hline $\mathrm{H} 15 \mathrm{C}$ & 0.1961 & 0.8357 & 0.9489 & $0.067^{*}$ & 0.4 \\
\hline H15D & 0.2903 & 0.9301 & 0.9348 & $0.067^{*}$ & 0.4 \\
\hline $\mathrm{C} 16 \mathrm{~A}$ & $0.394(2)$ & $0.851(4)$ & $0.9692(11)$ & $0.16(2)$ & 0.6 \\
\hline H16A & 0.4103 & 0.7700 & 0.9698 & $0.192 *$ & 0.6 \\
\hline H16B & 0.4560 & 0.8815 & 0.9473 & $0.192 *$ & 0.6 \\
\hline C17A & $0.400(2)$ & $0.926(3)$ & $1.0325(9)$ & $0.172(18)$ & 0.6 \\
\hline H17A & 0.3964 & 1.0084 & 1.0323 & $0.258 *$ & 0.6 \\
\hline H17B & 0.4719 & 0.9143 & 1.0513 & $0.258^{*}$ & 0.6 \\
\hline $\mathrm{H} 17 \mathrm{C}$ & 0.3340 & 0.9018 & 1.0538 & $0.258 *$ & 0.6 \\
\hline $\mathrm{C} 16 \mathrm{~B}$ & $0.367(2)$ & $0.811(3)$ & $0.9773(13)$ & $0.058(8)$ & 0.4 \\
\hline $\mathrm{H} 16 \mathrm{C}$ & 0.4381 & 0.7876 & 0.9565 & $0.069^{*}$ & 0.4 \\
\hline H16D & 0.3867 & 0.8774 & 1.0109 & $0.069^{*}$ & 0.4 \\
\hline C17B & $0.306(3)$ & $0.705(3)$ & $0.9983(12)$ & $0.086(9)$ & 0.4 \\
\hline H17D & 0.2298 & 0.7260 & 1.0136 & $0.130 *$ & 0.4 \\
\hline H17E & 0.3536 & 0.6868 & 1.0288 & $0.130^{*}$ & 0.4 \\
\hline $\mathrm{H} 17 \mathrm{~F}$ & 0.2965 & 0.6367 & 0.9654 & $0.130^{*}$ & 0.4 \\
\hline C18 & $0.2168(6)$ & $0.6875(6)$ & $0.7706(3)$ & $0.0341(13)$ & \\
\hline H18A & 0.2361 & 0.6105 & 0.7763 & $0.041^{*}$ & \\
\hline H18B & 0.2872 & 0.7241 & 0.7571 & $0.041^{*}$ & \\
\hline C19 & $0.1213(7)$ & $0.6679(7)$ & $0.7222(4)$ & $0.0442(17)$ & \\
\hline H19A & 0.0830 & 0.7414 & 0.7245 & $0.053^{*}$ & \\
\hline H19B & 0.0622 & 0.6076 & 0.7279 & $0.053 *$ & \\
\hline C20 & $0.1771(9)$ & $0.6273(9)$ & $0.6606(4)$ & $0.067(3)$ & \\
\hline
\end{tabular}




\begin{tabular}{|c|c|c|c|c|c|}
\hline $\mathrm{H} 20 \mathrm{~A}$ & 0.1148 & 0.5955 & 0.6312 & $0.080^{*}$ & \\
\hline $\mathrm{H} 20 \mathrm{~B}$ & 0.2281 & 0.5636 & 0.6615 & $0.080^{*}$ & \\
\hline C21 & $0.2499(11)$ & $0.7297(13)$ & $0.6413(5)$ & $0.088(4)$ & \\
\hline $\mathrm{H} 21 \mathrm{~A}$ & 0.2812 & 0.6998 & 0.6027 & $0.133^{*}$ & \\
\hline $\mathrm{H} 21 \mathrm{~B}$ & 0.3137 & 0.7596 & 0.6693 & $0.133^{*}$ & \\
\hline $\mathrm{H} 21 \mathrm{C}$ & 0.1999 & 0.7928 & 0.6400 & $0.133^{*}$ & \\
\hline $\mathrm{C} 22$ & $0.1610(6)$ & $0.8890(6)$ & $0.8236(3)$ & $0.0389(15)$ & \\
\hline $\mathrm{H} 22 \mathrm{~A}$ & 0.1364 & 0.9351 & 0.8616 & $0.047 *$ & \\
\hline $\mathrm{H} 22 \mathrm{~B}$ & 0.0957 & 0.8826 & 0.7956 & $0.047 *$ & \\
\hline $\mathrm{C} 23$ & $0.2649(7)$ & $0.9579(7)$ & $0.8031(4)$ & $0.051(2)$ & \\
\hline $\mathrm{H} 23 \mathrm{~A}$ & 0.3226 & 0.9837 & 0.8354 & $0.062 *$ & \\
\hline H23B & 0.3024 & 0.9058 & 0.7705 & $0.062 *$ & \\
\hline $\mathrm{C} 24$ & $0.2266(8)$ & $1.0655(7)$ & $0.7835(5)$ & $0.056(2)$ & \\
\hline $\mathrm{H} 24 \mathrm{~A}$ & 0.1927 & 1.1197 & 0.8167 & $0.067^{*}$ & \\
\hline $\mathrm{H} 24 \mathrm{~B}$ & 0.1663 & 1.0404 & 0.7525 & $0.067 *$ & \\
\hline $\mathrm{C} 25$ & $0.3294(10)$ & $1.1297(9)$ & $0.7607(6)$ & $0.075(3)$ & \\
\hline $\mathrm{H} 25 \mathrm{~A}$ & 0.3846 & 1.1634 & 0.7927 & $0.112 *$ & \\
\hline $\mathrm{H} 25 \mathrm{~B}$ & 0.3012 & 1.1922 & 0.7445 & $0.112^{*}$ & \\
\hline $\mathrm{H} 25 \mathrm{C}$ & 0.3676 & 1.0744 & 0.7303 & $0.112 *$ & \\
\hline $\mathrm{C} 26$ & $0.6939(6)$ & $0.2858(7)$ & 0.7279 & 0.0408 (16) & \\
\hline $\mathrm{H} 26 \mathrm{~A}$ & 0.6140 & 0.3061 & 0.7219 & $0.049 *$ & \\
\hline $\mathrm{H} 26 \mathrm{~B}$ & 0.7377 & 0.3579 & 0.7497 & $0.049^{*}$ & \\
\hline $\mathrm{C} 27$ & $0.6901(9)$ & 0.1948 (11) & $0.7660(4)$ & $0.072(3)$ & \\
\hline $\mathrm{H} 27 \mathrm{~A}$ & 0.7708 & 0.1779 & 0.7729 & $0.087^{*}$ & 0.4 \\
\hline $\mathrm{H} 27 \mathrm{~B}$ & 0.6509 & 0.1216 & 0.7426 & $0.087^{*}$ & 0.4 \\
\hline $\mathrm{H} 27 \mathrm{C}$ & 0.7676 & 0.1668 & 0.7704 & $0.087^{*}$ & 0.6 \\
\hline $\mathrm{H} 27 \mathrm{D}$ & 0.6354 & 0.1270 & 0.7493 & $0.087^{*}$ & 0.6 \\
\hline C28A & $0.630(2)$ & $0.224(2)$ & $0.8267(8)$ & $0.039(6)$ & 0.4 \\
\hline $\mathrm{H} 28 \mathrm{~A}$ & 0.5561 & 0.2594 & 0.8246 & $0.047^{*}$ & 0.4 \\
\hline $\mathrm{H} 28 \mathrm{~B}$ & 0.6172 & 0.1530 & 0.8421 & $0.047^{*}$ & 0.4 \\
\hline C29A & 0.7227 (19) & $0.312(2)$ & $0.8636(10)$ & $0.061(6)$ & 0.4 \\
\hline $\mathrm{H} 29 \mathrm{~A}$ & 0.7973 & 0.2768 & 0.8612 & $0.091 *$ & 0.4 \\
\hline H29B & 0.6991 & 0.3324 & 0.9042 & $0.091^{*}$ & 0.4 \\
\hline $\mathrm{H} 29 \mathrm{C}$ & 0.7300 & 0.3827 & 0.8487 & $0.091 *$ & 0.4 \\
\hline C28B & $0.6464(17)$ & $0.268(2)$ & $0.8264(7)$ & $0.080(9)$ & 0.6 \\
\hline $\mathrm{H} 28 \mathrm{C}$ & 0.7048 & 0.3318 & 0.8441 & $0.096^{*}$ & 0.6 \\
\hline $\mathrm{H} 28 \mathrm{D}$ & 0.5733 & 0.3034 & 0.8206 & $0.096^{*}$ & 0.6 \\
\hline C29B & $0.627(2)$ & $0.181(3)$ & 0.8667 (11) & $0.172(18)$ & 0.6 \\
\hline H29D & 0.5565 & 0.1306 & 0.8540 & $0.258 *$ & 0.6 \\
\hline $\mathrm{H} 29 \mathrm{E}$ & 0.6188 & 0.2251 & 0.9069 & $0.258 *$ & 0.6 \\
\hline $\mathrm{H} 29 \mathrm{~F}$ & 0.6932 & 0.1328 & 0.8644 & $0.258^{*}$ & 0.6 \\
\hline $\mathrm{C} 30$ & $0.8787(6)$ & $0.2231(6)$ & $0.6741(4)$ & $0.0404(16)$ & \\
\hline $\mathrm{H} 30 \mathrm{~A}$ & 0.8842 & 0.1626 & 0.6965 & $0.049 *$ & \\
\hline Н30B & 0.9086 & 0.1912 & 0.6350 & $0.049 *$ & \\
\hline C31 & $0.9577(7)$ & $0.3320(9)$ & $0.7044(5)$ & $0.069(3)$ & \\
\hline H31A & 0.9315 & 0.3628 & 0.7444 & $0.082 *$ & \\
\hline H31B & 0.9525 & 0.3940 & 0.6829 & $0.082 *$ & \\
\hline C32 & $1.0857(7)$ & $0.2986(9)$ & $0.7062(5)$ & $0.068(3)$ & \\
\hline
\end{tabular}




$\begin{array}{lllll}\text { H32A } & 1.1326 & 0.3662 & 0.7301 & 0.082^{*} \\ \text { H32B } & 1.0886 & 0.2334 & 0.7257 & 0.082^{*} \\ \text { C33 } & 1.1404(9) & 0.2630(11) & 0.6472(6) & 0.093(4) \\ \text { H33A } & 1.1033 & 0.1884 & 0.6255 & 0.139^{*} \\ \text { H33B } & 1.2231 & 0.2546 & 0.6524 & 0.139^{*} \\ \text { H33C } & 1.1303 & 0.3230 & 0.6256 & 0.139^{*} \\ \text { C34 } & 0.6902(6) & 0.1279(6) & 0.6335(3) & 0.0347(14) \\ \text { H34A } & 0.7049 & 0.0675 & 0.6549 & 0.042^{*} \\ \text { H34B } & 0.7279 & 0.1055 & 0.5954 & 0.042^{*} \\ \text { C35 } & 0.5576(6) & 0.1257(6) & 0.6223(3) & 0.0369(14) \\ \text { H35A } & 0.5188 & 0.1534 & 0.6597 & 0.044^{*} \\ \text { H35B } & 0.5417 & 0.1791 & 0.5971 & 0.044^{*} \\ \text { C36 } & 0.5090(7) & 0.0014(7) & 0.5931(4) & 0.0478(18) \\ \text { H36A } & 0.5227 & -0.0507 & 0.6192 & 0.057^{*} \\ \text { H36B } & 0.5513 & -0.0272 & 0.5569 & 0.057^{*} \\ \text { C37 } & 0.3771(7) & -0.0056(7) & 0.5782(4) & 0.052(2) \\ \text { H37A } & 0.3340 & 0.0151 & 0.6142 & 0.078^{*} \\ \text { H37B } & 0.3521 & -0.0852 & 0.5569 & 0.078^{*} \\ \text { H37C } & 0.3622 & 0.0491 & 0.5540 & 0.078^{*} \\ \text { C38 } & 0.7337(6) & 0.3428(6) & 0.6346(3) & 0.0383(15) \\ \text { H38A } & 0.7721 & 0.4167 & 0.6577 & 0.046^{*} \\ \text { H38B } & 0.6502 & 0.3545 & 0.6321 & 0.046^{*} \\ \text { C39 } & 0.7830(8) & 0.3177(8) & 0.5717(4) & 0.059(2) \\ \text { H39A } & 0.8636 & 0.2949 & 0.5728 & 0.071^{*} \\ \text { H39B } & 0.7364 & 0.2517 & 0.5463 & 0.071^{*} \\ \text { C40 } & 0.7805(8) & 0.4279(9) & 0.5459(5) & 0.068(3) \\ \text { H40A } & 0.7857 & 0.4021 & 0.5031 & 0.081^{*} \\ \text { H40B } & 0.7046 & 0.4613 & 0.5543 & 0.081^{*} \\ \text { C41 } & 0.8687(11) & 0.5185(12) & 0.5664(8) & 0.111(5) \\ \text { H41A } & 0.8663 & 0.5439 & 0.6088 & 0.166^{*} \\ \text { H41B } & 0.8560 & 0.5847 & 0.5493 & 0.166^{*} \\ \text { H41C } & 0.9443 & 0.4891 & 0.5550 & 0.166^{*} \\ \text { C42 } & 0.9468(8) & 0.0777(8) & 0.4729(3) & 0.053(2) \\ \text { H42A } & 0.9359 & 0.1585 & 0.4697 & 0.063^{*} \\ \text { H42B } & 0.8994 & 0.0234 & 0.4419 & 0.063^{*} \\ \text { C43 } & 0.9270(8) & -0.0530(7) & 0.5351(3) & 0.050(2) \\ \text { H43A } & 0.8791 & -0.1102 & 0.5054 & 0.060^{*} \\ \text { H43B } & 0.9027 & -0.0618 & 0.5739 & 0.060^{*} \\ & & & & \end{array}$

Atomic displacement parameters $\left(\AA^{2}\right)$

\begin{tabular}{lllllll}
\hline & $U^{11}$ & $U^{22}$ & $U^{33}$ & $U^{12}$ & $U^{13}$ & $U^{23}$ \\
\hline $\mathrm{I} 1$ & $0.0304(2)$ & $0.0365(2)$ & $0.0282(2)$ & $-0.01134(16)$ & $-0.00065(15)$ & $-0.00781(16)$ \\
$\mathrm{I} 2$ & $0.0451(2)$ & $0.02683(19)$ & $0.01655(16)$ & $-0.00402(16)$ & $-0.00845(14)$ & $0.00788(13)$ \\
$\mathrm{I} 3$ & $0.0544(3)$ & $0.0603(3)$ & $0.0397(3)$ & $-0.0281(2)$ & $0.0047(2)$ & $0.0250(2)$ \\
$\mathrm{Cu} 1$ & $0.0394(4)$ & $0.0449(5)$ & $0.0183(3)$ & $-0.0246(4)$ & $-0.0077(3)$ & $0.0133(3)$ \\
$\mathrm{Cu} 2$ & $0.0263(3)$ & $0.0242(3)$ & $0.0158(3)$ & $-0.0093(3)$ & $-0.0060(2)$ & $0.0080(2)$ \\
$\mathrm{Cu} 3$ & $0.0208(3)$ & $0.0208(3)$ & $0.0159(3)$ & $-0.0045(2)$ & $-0.0005(2)$ & $0.0046(2)$
\end{tabular}




\begin{tabular}{|c|c|c|c|c|c|c|}
\hline Cl1A & $0.057(2)$ & 0.078 (3) & $0.0226(14)$ & $-0.046(2)$ & $-0.0200(14)$ & 0.0287 (17) \\
\hline Cl1B & $0.057(3)$ & $0.058(3)$ & $0.022(2)$ & $-0.039(3)$ & $-0.020(2)$ & $0.021(2)$ \\
\hline $\mathrm{Cl} 2$ & $0.0199(5)$ & $0.0198(5)$ & $0.0127(5)$ & $-0.0122(4)$ & $-0.0068(4)$ & $0.0101(4)$ \\
\hline $\mathrm{Cl} 3$ & $0.0186(5)$ & $0.0145(5)$ & $0.0119(5)$ & $-0.0028(4)$ & $-0.0028(4)$ & $0.0022(4)$ \\
\hline $\mathrm{Cl} 4$ & $0.0096(5)$ & $0.0206(5)$ & $0.0122(5)$ & $-0.0027(4)$ & $0.0013(4)$ & $-0.0001(4)$ \\
\hline $\mathrm{Cl} 5$ & 0.0114 (4) & $0.0122(5)$ & 0.0115 (4) & $-0.0058(4)$ & $-0.0040(3)$ & 0.0075 (4) \\
\hline $\mathrm{O} 1$ & $0.046(3)$ & $0.051(3)$ & $0.042(3)$ & $0.014(2)$ & $0.018(2)$ & $0.013(2)$ \\
\hline N1 & $0.029(2)$ & $0.034(3)$ & $0.018(2)$ & $-0.012(2)$ & $-0.0026(18)$ & 0.0065 (19) \\
\hline N2 & $0.022(2)$ & $0.024(2)$ & 0.017 (2) & $-0.0027(17)$ & $-0.0029(16)$ & 0.0074 (17) \\
\hline N3 & $0.025(2)$ & $0.022(2)$ & $0.018(2)$ & $-0.0056(18)$ & $-0.0021(17)$ & $0.0081(17)$ \\
\hline N4 & $0.024(2)$ & $0.019(2)$ & 0.017 (2) & $-0.0049(17)$ & $-0.0018(16)$ & $0.0041(16)$ \\
\hline N5 & $0.022(2)$ & $0.027(2)$ & $0.016(2)$ & $-0.0041(18)$ & $-0.0009(16)$ & $0.0036(17)$ \\
\hline N6 & $0.028(2)$ & $0.034(3)$ & $0.019(2)$ & $-0.010(2)$ & $-0.0032(18)$ & 0.0087 (19) \\
\hline N7 & $0.020(2)$ & $0.039(3)$ & $0.043(3)$ & $0.002(2)$ & $0.003(2)$ & $0.010(2)$ \\
\hline N8 & $0.028(3)$ & $0.033(3)$ & $0.041(3)$ & $0.010(2)$ & $0.000(2)$ & $0.007(2)$ \\
\hline $\mathrm{C} 1$ & $0.022(2)$ & $0.020(2)$ & $0.023(3)$ & $-0.002(2)$ & $-0.004(2)$ & $0.005(2)$ \\
\hline $\mathrm{C} 2$ & $0.021(2)$ & $0.024(3)$ & $0.021(2)$ & $0.000(2)$ & $-0.0027(19)$ & $-0.002(2)$ \\
\hline C3 & $0.028(3)$ & $0.039(3)$ & $0.019(3)$ & $-0.013(2)$ & $-0.003(2)$ & $0.006(2)$ \\
\hline $\mathrm{C} 4$ & $0.027(3)$ & $0.022(3)$ & $0.020(2)$ & $-0.003(2)$ & $-0.009(2)$ & $0.008(2)$ \\
\hline $\mathrm{C} 5$ & $0.032(3)$ & $0.022(3)$ & $0.016(2)$ & $0.000(2)$ & $-0.005(2)$ & $0.005(2)$ \\
\hline C6 & $0.028(3)$ & $0.024(3)$ & $0.020(2)$ & $-0.002(2)$ & $-0.003(2)$ & $0.010(2)$ \\
\hline C7 & $0.033(3)$ & $0.036(3)$ & $0.022(3)$ & -0.009 (2) & $0.001(2)$ & $0.014(2)$ \\
\hline $\mathrm{C} 8$ & $0.036(3)$ & $0.038(3)$ & $0.022(3)$ & $-0.012(3)$ & $0.004(2)$ & $0.014(2)$ \\
\hline C9 & $0.024(3)$ & $0.030(3)$ & $0.023(3)$ & $-0.006(2)$ & $0.001(2)$ & $0.008(2)$ \\
\hline $\mathrm{C} 10$ & $0.026(3)$ & $0.091(7)$ & $0.059(5)$ & $-0.008(4)$ & $0.001(3)$ & $0.038(5)$ \\
\hline C11 & $0.046(5)$ & $0.138(11)$ & $0.168(13)$ & $-0.027(6)$ & $-0.026(7)$ & 0.125 (11) \\
\hline $\mathrm{C} 12 \mathrm{~A}$ & $0.039(7)$ & $0.059(9)$ & $0.052(8)$ & $-0.011(6)$ & $-0.011(6)$ & $0.036(7)$ \\
\hline $\mathrm{C} 13 \mathrm{~A}$ & $0.081(13)$ & 0.119 (18) & $0.18(2)$ & $-0.053(13)$ & $-0.055(14)$ & $0.13(2)$ \\
\hline $\mathrm{C} 12 \mathrm{~B}$ & $0.08(2)$ & $0.08(2)$ & $0.054(15)$ & $-0.053(18)$ & $-0.005(13)$ & $0.006(14)$ \\
\hline C13B & $0.12(3)$ & 0.077 (19) & 0.057 (16) & $0.021(19)$ & $0.001(16)$ & $0.002(14)$ \\
\hline C14 & $0.027(3)$ & $0.047(4)$ & $0.031(3)$ & $0.005(3)$ & $0.002(2)$ & $0.001(3)$ \\
\hline $\mathrm{C} 15$ & $0.046(4)$ & $0.074(6)$ & $0.039(4)$ & $0.014(4)$ & $0.007(3)$ & $-0.005(4)$ \\
\hline $\mathrm{C} 16 \mathrm{~A}$ & $0.23(4)$ & $0.18(4)$ & $0.046(14)$ & $0.15(3)$ & $-0.06(2)$ & $-0.043(18)$ \\
\hline C17A & 0.077 (15) & $0.33(5)$ & $0.062(13)$ & $0.07(2)$ & $-0.019(11)$ & $-0.06(2)$ \\
\hline $\mathrm{C} 16 \mathrm{~B}$ & 0.067 (16) & $0.071(18)$ & 0.024 (11) & $-0.005(14)$ & $-0.015(10)$ & $-0.008(10)$ \\
\hline C17B & $0.071(17)$ & $0.12(3)$ & $0.053(15)$ & $0.003(17)$ & 0.008 (13) & $0.001(16)$ \\
\hline $\mathrm{C} 18$ & $0.033(3)$ & $0.029(3)$ & $0.040(3)$ & $0.006(3)$ & -0.004 & $0.008(3)$ \\
\hline C19 & $0.041(4)$ & 0.037 (4) & $0.054(4)$ & $0.003(3)$ & -0.019 (3) & 0.010 (3) \\
\hline $\mathrm{C} 20$ & $0.073(6)$ & $0.068(6)$ & $0.049(5)$ & $0.033(5)$ & $-0.029(4)$ & -0.013 \\
\hline $\mathrm{C} 21$ & $0.081(8)$ & $0.139(12)$ & $0.051(6)$ & $0.033(8)$ & $0.012(5)$ & $0.026(7)$ \\
\hline $\mathrm{C} 22$ & $0.027(3)$ & $0.035(3)$ & $0.053(4)$ & $0.010(3)$ & $0.003(3)$ & $0.004(3)$ \\
\hline $\mathrm{C} 23$ & $0.034(4)$ & 0.033 (4) & $0.086(6)$ & 0.005 (3) & 0.003 (4) & 0.008 (4) \\
\hline $\mathrm{C} 24$ & $0.052(5)$ & $0.034(4)$ & $0.080(6)$ & $-0.002(3)$ & $-0.006(4)$ & $0.011(4)$ \\
\hline $\mathrm{C} 25$ & $0.071(7)$ & $0.046(5)$ & $0.109(9)$ & $-0.009(5)$ & 0.007 (6) & $0.024(5)$ \\
\hline $\mathrm{C} 26$ & $0.034(3)$ & $0.050(4)$ & $0.036(3)$ & $0.016(3)$ & $-0.001(3)$ & $0.002(3)$ \\
\hline C27 & $0.058(5)$ & 0.125 (9) & 0.048 (5) & $0.058(6)$ & $0.011(4)$ & 0.035 (5) \\
\hline $\mathrm{C} 28 \mathrm{~A}$ & 0.047 (13) & $0.032(9)$ & $0.031(11)$ & $-0.017(8)$ & $-0.004(8)$ & $-0.005(8)$ \\
\hline $\mathrm{C} 29 \mathrm{~A}$ & 0.054 (13) & $0.072(15)$ & 0.052 (12) & $0.010(11)$ & $-0.003(10)$ & 0.003 (11) \\
\hline
\end{tabular}




\begin{tabular}{lllllll} 
C28B & $0.025(8)$ & $0.18(3)$ & $0.054(12)$ & $0.036(13)$ & $0.009(7)$ & $0.048(14)$ \\
C29B & $0.14(2)$ & $0.34(4)$ & $0.121(18)$ & $0.19(3)$ & $0.102(17)$ & $0.17(3)$ \\
C30 & $0.024(3)$ & $0.037(4)$ & $0.057(4)$ & $0.015(3)$ & $0.002(3)$ & $0.001(3)$ \\
C31 & $0.032(4)$ & $0.061(5)$ & $0.092(7)$ & $0.013(4)$ & $-0.015(4)$ & $-0.024(5)$ \\
C32 & $0.031(4)$ & $0.060(6)$ & $0.103(8)$ & $0.011(4)$ & $-0.010(4)$ & $-0.003(5)$ \\
C33 & $0.040(5)$ & $0.080(7)$ & $0.125(10)$ & $-0.017(5)$ & $0.012(6)$ & $-0.037(7)$ \\
C34 & $0.039(3)$ & $0.026(3)$ & $0.039(3)$ & $0.010(3)$ & $-0.002(3)$ & $0.006(3)$ \\
C35 & $0.038(3)$ & $0.029(3)$ & $0.047(4)$ & $0.007(3)$ & $-0.001(3)$ & $0.013(3)$ \\
C36 & $0.045(4)$ & $0.033(4)$ & $0.061(5)$ & $0.008(3)$ & $-0.003(4)$ & $0.002(3)$ \\
C37 & $0.046(4)$ & $0.045(4)$ & $0.064(5)$ & $-0.004(3)$ & $-0.011(4)$ & $0.015(4)$ \\
C38 & $0.032(3)$ & $0.025(3)$ & $0.060(4)$ & $0.011(3)$ & $0.004(3)$ & $0.013(3)$ \\
C39 & $0.061(5)$ & $0.057(5)$ & $0.070(6)$ & $0.030(4)$ & $0.020(4)$ & $0.033(5)$ \\
C40 & $0.049(5)$ & $0.064(6)$ & $0.106(8)$ & $0.017(4)$ & $0.008(5)$ & $0.047(6)$ \\
C41 & $0.068(8)$ & $0.079(8)$ & $0.200(18)$ & $0.003(6)$ & $-0.021(9)$ & $0.063(10)$ \\
C42 & $0.075(6)$ & $0.052(5)$ & $0.038(4)$ & $0.026(4)$ & $0.007(4)$ & $0.019(3)$ \\
C43 & $0.066(5)$ & $0.045(4)$ & $0.039(4)$ & $-0.013(4)$ & $0.019(4)$ & $0.014(3)$ \\
& & & & & & \\
\hline
\end{tabular}

Geometric parameters $\left(\AA,{ }^{\circ}\right)$

\begin{tabular}{llll}
\hline $\mathrm{I} 1-\mathrm{C} 2$ & $2.063(5)$ & $\mathrm{C} 17 \mathrm{~B}-\mathrm{H} 17 \mathrm{E}$ & 0.9600 \\
$\mathrm{I} 2-\mathrm{C} 5$ & $2.069(5)$ & $\mathrm{C} 17 \mathrm{~B}-\mathrm{H} 17 \mathrm{~F}$ & 0.9600 \\
$\mathrm{I} 3-\mathrm{C} 8$ & $2.060(6)$ & $\mathrm{C} 18-\mathrm{C} 19$ & $1.523(9)$ \\
$\mathrm{Cu} 1-\mathrm{N} 1$ & $1.926(5)$ & $\mathrm{C} 18-\mathrm{H} 18 \mathrm{~A}$ & 0.9700 \\
$\mathrm{Cu} 1-\mathrm{N} 6$ & $1.926(5)$ & $\mathrm{C} 18-\mathrm{H} 18 \mathrm{~B}$ & 0.9700 \\
$\mathrm{Cu} 1-\mathrm{C} 11 \mathrm{~A}$ & $2.289(3)$ & $\mathrm{C} 19-\mathrm{C} 20$ & $1.550(13)$ \\
$\mathrm{Cu} 1-\mathrm{C} 11 \mathrm{~B}$ & $2.335(5)$ & $\mathrm{C} 19-\mathrm{H} 19 \mathrm{~A}$ & 0.9700 \\
$\mathrm{Cu} 1-\mathrm{C} 14$ & $2.6258(14)$ & $\mathrm{C} 19-\mathrm{H} 19 \mathrm{~B}$ & 0.9700 \\
$\mathrm{Cu} 1-\mathrm{C} 15$ & $2.6478(14)$ & $\mathrm{C} 20-\mathrm{C} 21$ & $1.555(17)$ \\
$\mathrm{Cu} 2-\mathrm{N} 2$ & $1.933(4)$ & $\mathrm{C} 20-\mathrm{H} 20 \mathrm{~A}$ & 0.9700 \\
$\mathrm{Cu} 2-\mathrm{N} 3$ & $1.936(4)$ & $\mathrm{C} 20-\mathrm{H} 20 \mathrm{~B}$ & 0.9700 \\
$\mathrm{Cu} 2-\mathrm{C} 2$ & $2.3458(12)$ & $\mathrm{C} 21-\mathrm{H} 21 \mathrm{~A}$ & 0.9600 \\
$\mathrm{Cu} 2-\mathrm{C} 15$ & $2.5808(12)$ & $\mathrm{C} 21-\mathrm{H} 21 \mathrm{~B}$ & 0.9600 \\
$\mathrm{Cu} 2-\mathrm{C} 14$ & $2.6306(13)$ & $\mathrm{C} 21-\mathrm{H} 21 \mathrm{C}$ & 0.9600 \\
$\mathrm{Cu} 3-\mathrm{N} 4$ & $1.945(4)$ & $\mathrm{C} 22-\mathrm{C} 23$ & $1.524(10)$ \\
$\mathrm{Cu} 3-\mathrm{N} 5$ & $1.951(5)$ & $\mathrm{C} 22-\mathrm{H} 22 \mathrm{~A}$ & 0.9700 \\
$\mathrm{Cu} 3-\mathrm{C} 13$ & $2.3337(12)$ & $\mathrm{C} 22-\mathrm{H} 22 \mathrm{~B}$ & 0.9700 \\
$\mathrm{Cu} 3-\mathrm{C} 14$ & $2.5016(13)$ & $\mathrm{C} 23-\mathrm{C} 24$ & $1.510(11)$ \\
$\mathrm{Cu} 3-\mathrm{C} 15$ & $2.7607(12)$ & $\mathrm{C} 23-\mathrm{H} 23 \mathrm{~A}$ & 0.9700 \\
$\mathrm{O} 1-\mathrm{C} 43$ & $1.412(10)$ & $\mathrm{C} 23-\mathrm{H} 23 \mathrm{~B}$ & 0.9700 \\
$\mathrm{O} 1-\mathrm{C} 42$ & $1.433(9)$ & $\mathrm{C} 24-\mathrm{C} 25$ & $1.510(13)$ \\
$\mathrm{N} 1-\mathrm{N} 2$ & $1.347(6)$ & $\mathrm{C} 24-\mathrm{H} 24 \mathrm{~A}$ & 0.9700 \\
$\mathrm{~N} 1-\mathrm{C} 3$ & $1.353(7)$ & $\mathrm{C} 24-\mathrm{H} 24 \mathrm{~B}$ & 0.9700 \\
$\mathrm{~N} 2-\mathrm{C} 1$ & $1.356(7)$ & $\mathrm{C} 25-\mathrm{H} 25 \mathrm{~A}$ & 0.9600 \\
$\mathrm{~N} 3-\mathrm{C} 6$ & $1.337(7)$ & $\mathrm{C} 25-\mathrm{H} 25 \mathrm{~B}$ & 0.9600 \\
$\mathrm{~N} 3-\mathrm{N} 4$ & $\mathrm{C} 25-\mathrm{H} 25 \mathrm{C}$ & 0.9600 \\
$\mathrm{~N} 4-\mathrm{C} 4$ & $\mathrm{C} 26-\mathrm{C} 27$ & 0.9700 \\
$\mathrm{~N} 5-\mathrm{C} 9$ & $\mathrm{C} 26-\mathrm{H} 26 \mathrm{~A}$ & 0.9700 \\
$\mathrm{~N} 5-\mathrm{N} 6$ & $1.364(6)$ & $\mathrm{C} 26-\mathrm{H} 26 \mathrm{~B}$ &
\end{tabular}




\begin{tabular}{|c|c|c|c|}
\hline N6-C7 & $1.344(7)$ & $\mathrm{C} 27-\mathrm{C} 28 \mathrm{~A}$ & $1.542(17)$ \\
\hline $\mathrm{N} 7-\mathrm{C} 22$ & $1.516(9)$ & $\mathrm{C} 27-\mathrm{C} 28 \mathrm{~B}$ & $1.560(16)$ \\
\hline $\mathrm{N} 7-\mathrm{C} 18$ & $1.518(9)$ & $\mathrm{C} 27-\mathrm{H} 27 \mathrm{~A}$ & 0.9700 \\
\hline $\mathrm{N} 7-\mathrm{C} 10$ & $1.519(9)$ & $\mathrm{C} 27-\mathrm{H} 27 \mathrm{~B}$ & 0.9700 \\
\hline $\mathrm{N} 7-\mathrm{C} 14$ & $1.521(8)$ & $\mathrm{C} 27-\mathrm{H} 27 \mathrm{C}$ & 0.9700 \\
\hline $\mathrm{N} 8-\mathrm{C} 34$ & $1.517(8)$ & $\mathrm{C} 27-\mathrm{H} 27 \mathrm{D}$ & 0.9700 \\
\hline $\mathrm{N} 8-\mathrm{C} 26$ & $1.517(9)$ & $\mathrm{C} 28 \mathrm{~A}-\mathrm{C} 29 \mathrm{~A}$ & $1.522(17)$ \\
\hline $\mathrm{N} 8-\mathrm{C} 38$ & $1.523(9)$ & $\mathrm{C} 28 \mathrm{~A}-\mathrm{H} 28 \mathrm{~A}$ & 0.9600 \\
\hline $\mathrm{N} 8-\mathrm{C} 30$ & $1.523(8)$ & $\mathrm{C} 28 \mathrm{~A}-\mathrm{H} 28 \mathrm{~B}$ & 0.9700 \\
\hline $\mathrm{C} 1-\mathrm{C} 2$ & $1.394(7)$ & $\mathrm{C} 29 \mathrm{~A}-\mathrm{H} 29 \mathrm{~A}$ & 0.9600 \\
\hline $\mathrm{C} 1-\mathrm{H} 1$ & 0.9300 & $\mathrm{C} 29 \mathrm{~A}-\mathrm{H} 29 \mathrm{~B}$ & 0.9600 \\
\hline $\mathrm{C} 2-\mathrm{C} 3$ & $1.382(8)$ & $\mathrm{C} 29 \mathrm{~A}-\mathrm{H} 29 \mathrm{C}$ & 0.9600 \\
\hline $\mathrm{C} 3-\mathrm{H} 3$ & 0.9300 & $\mathrm{C} 28 \mathrm{~B}-\mathrm{C} 29 \mathrm{~B}$ & $1.538(18)$ \\
\hline $\mathrm{C} 4-\mathrm{C} 5$ & $1.387(8)$ & $\mathrm{C} 28 \mathrm{~B}-\mathrm{H} 28 \mathrm{C}$ & 0.9700 \\
\hline $\mathrm{C} 4-\mathrm{H} 4$ & 0.9300 & $\mathrm{C} 28 \mathrm{~B}-\mathrm{H} 28 \mathrm{D}$ & 0.9700 \\
\hline $\mathrm{C} 5-\mathrm{C} 6$ & $1.381(7)$ & $\mathrm{C} 29 \mathrm{~B}-\mathrm{H} 29 \mathrm{D}$ & 0.9600 \\
\hline $\mathrm{C} 6-\mathrm{H} 6$ & 0.9300 & $\mathrm{C} 29 \mathrm{~B}-\mathrm{H} 29 \mathrm{E}$ & 0.9600 \\
\hline $\mathrm{C} 7-\mathrm{C} 8$ & $1.389(8)$ & $\mathrm{C} 29 \mathrm{~B}-\mathrm{H} 29 \mathrm{~F}$ & 0.9600 \\
\hline $\mathrm{C} 7-\mathrm{H} 7$ & 0.9300 & $\mathrm{C} 30-\mathrm{C} 31$ & $1.521(11)$ \\
\hline $\mathrm{C} 8-\mathrm{C} 9$ & $1.401(8)$ & $\mathrm{C} 30-\mathrm{H} 30 \mathrm{~A}$ & 0.9700 \\
\hline C9-H9 & 0.9300 & $\mathrm{C} 30-\mathrm{H} 30 \mathrm{~B}$ & 0.9700 \\
\hline $\mathrm{C} 10-\mathrm{C} 11$ & $1.506(14)$ & C31-C32 & $1.531(11)$ \\
\hline C10-H10A & 0.9700 & $\mathrm{C} 31-\mathrm{H} 31 \mathrm{~A}$ & 0.9700 \\
\hline $\mathrm{C} 10-\mathrm{H} 10 \mathrm{~B}$ & 0.9700 & $\mathrm{C} 31-\mathrm{H} 31 \mathrm{~B}$ & 0.9700 \\
\hline $\mathrm{C} 11-\mathrm{C} 12 \mathrm{~B}$ & $1.514(16)$ & $\mathrm{C} 32-\mathrm{C} 33$ & $1.486(16)$ \\
\hline $\mathrm{C} 11-\mathrm{C} 12 \mathrm{~A}$ & $1.567(14)$ & $\mathrm{C} 32-\mathrm{H} 32 \mathrm{~A}$ & 0.9700 \\
\hline $\mathrm{C} 11-\mathrm{H} 11 \mathrm{~A}$ & 0.9700 & $\mathrm{C} 32-\mathrm{H} 32 \mathrm{~B}$ & 0.9700 \\
\hline C11-H11B & 0.9700 & $\mathrm{C} 33-\mathrm{H} 33 \mathrm{~A}$ & 0.9600 \\
\hline $\mathrm{C} 11-\mathrm{H} 11 \mathrm{C}$ & 0.9700 & C $33-\mathrm{H} 33 \mathrm{~B}$ & 0.9600 \\
\hline C11-H11D & 0.9700 & $\mathrm{C} 33-\mathrm{H} 33 \mathrm{C}$ & 0.9600 \\
\hline $\mathrm{C} 12 \mathrm{~A}-\mathrm{C} 13 \mathrm{~A}$ & $1.592(15)$ & $\mathrm{C} 34-\mathrm{C} 35$ & $1.525(9)$ \\
\hline $\mathrm{C} 12 \mathrm{~A}-\mathrm{H} 12 \mathrm{~A}$ & 0.9700 & $\mathrm{C} 34-\mathrm{H} 34 \mathrm{~A}$ & 0.9700 \\
\hline $\mathrm{C} 12 \mathrm{~A}-\mathrm{H} 12 \mathrm{~B}$ & 0.9700 & C34-H34B & 0.9700 \\
\hline $\mathrm{C} 13 \mathrm{~A}-\mathrm{H} 13 \mathrm{~A}$ & 0.9600 & C35-C36 & $1.504(10)$ \\
\hline $\mathrm{C} 13 \mathrm{~A}-\mathrm{H} 13 \mathrm{~B}$ & 0.9600 & $\mathrm{C} 35-\mathrm{H} 35 \mathrm{~A}$ & 0.9700 \\
\hline $\mathrm{C} 13 \mathrm{~A}-\mathrm{H} 13 \mathrm{C}$ & 0.9600 & $\mathrm{C} 35-\mathrm{H} 35 \mathrm{~B}$ & 0.9700 \\
\hline $\mathrm{C} 12 \mathrm{~B}-\mathrm{C} 13 \mathrm{~B}$ & $1.533(18)$ & $\mathrm{C} 36-\mathrm{C} 37$ & $1.529(11)$ \\
\hline $\mathrm{C} 12 \mathrm{~B}-\mathrm{H} 12 \mathrm{C}$ & 0.9700 & $\mathrm{C} 36-\mathrm{H} 36 \mathrm{~A}$ & 0.9700 \\
\hline $\mathrm{C} 12 \mathrm{~B}-\mathrm{H} 12 \mathrm{D}$ & 0.9600 & $\mathrm{C} 36-\mathrm{H} 36 \mathrm{~B}$ & 0.9700 \\
\hline $\mathrm{C} 13 \mathrm{~B}-\mathrm{H} 13 \mathrm{D}$ & 0.9600 & C37-H37A & 0.9600 \\
\hline $\mathrm{C} 13 \mathrm{~B}-\mathrm{H} 13 \mathrm{E}$ & 0.9600 & C37-H37B & 0.9600 \\
\hline $\mathrm{C} 13 \mathrm{~B}-\mathrm{H} 13 \mathrm{~F}$ & 0.9600 & $\mathrm{C} 37-\mathrm{H} 37 \mathrm{C}$ & 0.9600 \\
\hline $\mathrm{C} 14-\mathrm{C} 15$ & $1.522(10)$ & C38-C39 & $1.538(11)$ \\
\hline $\mathrm{C} 14-\mathrm{H} 14 \mathrm{~A}$ & 0.9700 & C38-H38A & 0.9700 \\
\hline C14-H14B & 0.9700 & C $38-H 38 B$ & 0.9700 \\
\hline $\mathrm{C} 15-\mathrm{C} 16 \mathrm{~A}$ & $1.548(17)$ & C39-C40 & $1.534(12)$ \\
\hline $\mathrm{C} 15-\mathrm{C} 16 \mathrm{~B}$ & $1.561(18)$ & $\mathrm{C} 39-\mathrm{H} 39 \mathrm{~A}$ & 0.9700 \\
\hline $\mathrm{C} 15-\mathrm{H} 15 \mathrm{~A}$ & 0.9700 & С $39-\mathrm{H} 39 \mathrm{~B}$ & 0.9700 \\
\hline
\end{tabular}




$\begin{array}{ll}\text { C15-H15B } & 0.9700 \\ \text { C15-H15C } & 0.9700 \\ \text { C15-H15D } & 0.9700 \\ \text { C16A-C17A } & 1.517(18) \\ \text { C16A-H16A } & 0.9700 \\ \text { C16A-H16B } & 0.9700 \\ \text { C17A-H17A } & 0.9600 \\ \text { C17A-H17B } & 0.9600 \\ \text { C17A-H17C } & 0.9600 \\ \text { C16B-C17B } & 1.548(19) \\ \text { C16B-H16C } & 0.9600 \\ \text { C16B-H16D } & 0.9700 \\ \text { C17B-H17D } & 0.9600\end{array}$

$\mathrm{N} 1-\mathrm{Cu} 1-\mathrm{N} 6$

$\mathrm{N} 1-\mathrm{Cu} 1-\mathrm{Cl1A}$

N6-Cu1-Cl1A

N1-Cu1-Cl1B

N6-Cu1-Cl1B

$\mathrm{N} 1-\mathrm{Cu} 1-\mathrm{Cl} 4$

$\mathrm{N} 6-\mathrm{Cu} 1-\mathrm{Cl} 4$

$\mathrm{Cl1} A-\mathrm{Cu} 1-\mathrm{Cl} 4$

$\mathrm{Cl1B}-\mathrm{Cu} 1-\mathrm{Cl} 4$

$\mathrm{N} 1-\mathrm{Cu} 1-\mathrm{Cl} 5$

$\mathrm{N} 6-\mathrm{Cu} 1-\mathrm{Cl} 5$

$\mathrm{C} 11 \mathrm{~A}-\mathrm{Cu} 1-\mathrm{Cl} 5$

$\mathrm{Cl1B}-\mathrm{Cu} 1-\mathrm{Cl} 5$

$\mathrm{Cl} 4-\mathrm{Cu} 1-\mathrm{Cl} 5$

$\mathrm{N} 2-\mathrm{Cu} 2-\mathrm{N} 3$

$\mathrm{N} 2-\mathrm{Cu} 2-\mathrm{Cl} 2$

$\mathrm{N} 3-\mathrm{Cu} 2-\mathrm{Cl} 2$

$\mathrm{N} 2-\mathrm{Cu} 2-\mathrm{Cl} 5$

$\mathrm{N} 3-\mathrm{Cu} 2-\mathrm{Cl} 5$

$\mathrm{Cl} 2-\mathrm{Cu} 2-\mathrm{Cl} 5$

$\mathrm{N} 2-\mathrm{Cu} 2-\mathrm{Cl} 4$

$\mathrm{N} 3-\mathrm{Cu} 2-\mathrm{Cl} 4$

$\mathrm{Cl} 2-\mathrm{Cu} 2-\mathrm{Cl} 4$

$\mathrm{Cl} 5-\mathrm{Cu} 2-\mathrm{Cl} 4$

$\mathrm{N} 4-\mathrm{Cu} 3-\mathrm{N} 5$

$\mathrm{N} 4-\mathrm{Cu} 3-\mathrm{Cl} 3$

$\mathrm{N} 5-\mathrm{Cu} 3-\mathrm{Cl} 3$

$\mathrm{N} 4-\mathrm{Cu} 3-\mathrm{Cl} 4$

$\mathrm{N} 5-\mathrm{Cu} 3-\mathrm{Cl} 4$

$\mathrm{Cl} 3-\mathrm{Cu} 3-\mathrm{Cl} 4$

$\mathrm{N} 4-\mathrm{Cu} 3-\mathrm{Cl} 5$

$\mathrm{N} 5-\mathrm{Cu} 3-\mathrm{Cl} 5$

$\mathrm{Cl} 3-\mathrm{Cu} 3-\mathrm{Cl} 5$

$\mathrm{Cl} 4-\mathrm{Cu} 3-\mathrm{Cl} 5$
$173.7(2)$

90.85 (16)

92.47 (16)

$93.60(18)$

91.57 (18)

$86.25(15)$

87.74 (15)

$140.10(15)$

163.21 (19)

$90.42(15)$

90.84 (16)

$136.96(15)$

$113.90(19)$

82.89 (4)

$170.5(2)$

$92.12(14)$

93.63 (13)

92.75 (14)

$90.56(14)$

$122.39(5)$

85.89 (14)

$85.60(14)$

$153.50(5)$

84.10 (4)

176.18 (19)

93.20 (13)

90.59 (14)

86.82 (14)

90.04 (14)

$151.28(5)$

88.00 (14)

89.45 (14)

$125.75(5)$

82.96 (4)

\section{$\mathrm{C} 40-\mathrm{C} 41$}

$\mathrm{C} 40-\mathrm{H} 40 \mathrm{~A}$

$\mathrm{C} 40-\mathrm{H} 40 \mathrm{~B}$

C41-H41A

C41-H41B

C41-H41C

$\mathrm{C} 42-\mathrm{C}_{4} 3^{\mathrm{i}}$

$\mathrm{C} 42-\mathrm{H} 42 \mathrm{~A}$

C42-H42B

$\mathrm{C} 43-\mathrm{C} 42^{\mathrm{i}}$

$\mathrm{C} 43-\mathrm{H} 43 \mathrm{~A}$

C43-H43B

H17D-C17B-H17E

C16B-C17B-H17F

H17D-C17B-H17F

H17E-C17B-H17F

N7-C18-C19

N7-C18-H18A

C19-C18-H18A

N7-C18-H18B

C19-C18-H18B

$\mathrm{H} 18 \mathrm{~A}-\mathrm{C} 18-\mathrm{H} 18 \mathrm{~B}$

C18-C19-C20

C18-C19-H19A

C20-C19-H19A

C18-C19-H19B

C20-C19-H19B

H19A-C19-H19B

C19-C20-C21

C19-C $20-\mathrm{H} 20 \mathrm{~A}$

$\mathrm{C} 21-\mathrm{C} 20-\mathrm{H} 20 \mathrm{~A}$

$\mathrm{C} 19-\mathrm{C} 20-\mathrm{H} 20 \mathrm{~B}$

$\mathrm{C} 21-\mathrm{C} 20-\mathrm{H} 20 \mathrm{~B}$

$\mathrm{H} 20 \mathrm{~A}-\mathrm{C} 20-\mathrm{H} 20 \mathrm{~B}$

$\mathrm{C} 20-\mathrm{C} 21-\mathrm{H} 21 \mathrm{~A}$

$\mathrm{C} 20-\mathrm{C} 21-\mathrm{H} 21 \mathrm{~B}$

$\mathrm{H} 21 \mathrm{~A}-\mathrm{C} 21-\mathrm{H} 21 \mathrm{~B}$

$\mathrm{C} 20-\mathrm{C} 21-\mathrm{H} 21 \mathrm{C}$

$\mathrm{H} 21 \mathrm{~A}-\mathrm{C} 21-\mathrm{H} 21 \mathrm{C}$

$\mathrm{H} 21 \mathrm{~B}-\mathrm{C} 21-\mathrm{H} 21 \mathrm{C}$

$\mathrm{N} 7-\mathrm{C} 22-\mathrm{C} 23$

$\mathrm{N} 7-\mathrm{C} 22-\mathrm{H} 22 \mathrm{~A}$

$\mathrm{C} 23-\mathrm{C} 22-\mathrm{H} 22 \mathrm{~A}$

$\mathrm{N} 7-\mathrm{C} 22-\mathrm{H} 22 \mathrm{~B}$

$\mathrm{C} 23-\mathrm{C} 22-\mathrm{H} 22 \mathrm{~B}$

$\mathrm{H} 22 \mathrm{~A}-\mathrm{C} 22-\mathrm{H} 22 \mathrm{~B}$
$1.397(16)$

0.9700

0.9700

0.9600

0.9600

0.9600

1.484 (12)

0.9700

0.9700

1.484 (12)

0.9700

0.9700

109.5

109.5

109.5

109.5

114.6 (6)

108.6

108.6

108.6

108.6

107.6

109.7 (6)

109.7

109.7

109.7

109.7

108.2

113.1 (8)

109.0

109.0

109.0

109.0

107.8

109.5

109.5

109.5

109.5

109.5

109.5

115.8 (5)

108.3

108.3

108.3

108.3

107.4 


\begin{tabular}{|c|c|c|c|}
\hline $\mathrm{Cu} 3-\mathrm{Cl} 4-\mathrm{Cu} 1$ & $82.74(4)$ & $\mathrm{C} 24-\mathrm{C} 23-\mathrm{C} 22$ & $111.7(6)$ \\
\hline $\mathrm{Cu} 3-\mathrm{Cl} 4-\mathrm{Cu} 2$ & $83.50(4)$ & $\mathrm{C} 24-\mathrm{C} 23-\mathrm{H} 23 \mathrm{~A}$ & 109.3 \\
\hline $\mathrm{Cu} 1-\mathrm{Cl} 4-\mathrm{Cu} 2$ & $79.97(4)$ & $\mathrm{C} 22-\mathrm{C} 23-\mathrm{H} 23 \mathrm{~A}$ & 109.3 \\
\hline $\mathrm{Cu} 2-\mathrm{Cl} 5-\mathrm{Cu} 1$ & $80.47(4)$ & $\mathrm{C} 24-\mathrm{C} 23-\mathrm{H} 23 \mathrm{~B}$ & 109.3 \\
\hline $\mathrm{Cu} 2-\mathrm{Cl} 5-\mathrm{Cu} 3$ & $79.51(3)$ & $\mathrm{C} 22-\mathrm{C} 23-\mathrm{H} 23 \mathrm{~B}$ & 109.3 \\
\hline $\mathrm{Cu} 1-\mathrm{Cl} 5-\mathrm{Cu} 3$ & $77.60(4)$ & $\mathrm{H} 23 \mathrm{~A}-\mathrm{C} 23-\mathrm{H} 23 \mathrm{~B}$ & 107.9 \\
\hline $\mathrm{C} 43-\mathrm{O} 1-\mathrm{C} 42$ & $109.8(6)$ & $\mathrm{C} 23-\mathrm{C} 24-\mathrm{C} 25$ & $111.2(8)$ \\
\hline $\mathrm{N} 2-\mathrm{N} 1-\mathrm{C} 3$ & $108.4(5)$ & $\mathrm{C} 23-\mathrm{C} 24-\mathrm{H} 24 \mathrm{~A}$ & 109.4 \\
\hline $\mathrm{N} 2-\mathrm{N} 1-\mathrm{Cu} 1$ & $122.7(4)$ & $\mathrm{C} 25-\mathrm{C} 24-\mathrm{H} 24 \mathrm{~A}$ & 109.4 \\
\hline $\mathrm{C} 3-\mathrm{N} 1-\mathrm{Cu} 1$ & $128.8(4)$ & $\mathrm{C} 23-\mathrm{C} 24-\mathrm{H} 24 \mathrm{~B}$ & 109.4 \\
\hline $\mathrm{N} 1-\mathrm{N} 2-\mathrm{C} 1$ & $108.5(4)$ & $\mathrm{C} 25-\mathrm{C} 24-\mathrm{H} 24 \mathrm{~B}$ & 109.4 \\
\hline $\mathrm{N} 1-\mathrm{N} 2-\mathrm{Cu} 2$ & $120.7(3)$ & $\mathrm{H} 24 \mathrm{~A}-\mathrm{C} 24-\mathrm{H} 24 \mathrm{~B}$ & 108.0 \\
\hline $\mathrm{C} 1-\mathrm{N} 2-\mathrm{Cu} 2$ & $130.7(4)$ & $\mathrm{C} 24-\mathrm{C} 25-\mathrm{H} 25 \mathrm{~A}$ & 109.5 \\
\hline $\mathrm{C} 6-\mathrm{N} 3-\mathrm{N} 4$ & $108.1(4)$ & $\mathrm{C} 24-\mathrm{C} 25-\mathrm{H} 25 \mathrm{~B}$ & 109.5 \\
\hline $\mathrm{C} 6-\mathrm{N} 3-\mathrm{Cu} 2$ & $129.9(4)$ & $\mathrm{H} 25 \mathrm{~A}-\mathrm{C} 25-\mathrm{H} 25 \mathrm{~B}$ & 109.5 \\
\hline $\mathrm{N} 4-\mathrm{N} 3-\mathrm{Cu} 2$ & $122.0(3)$ & $\mathrm{C} 24-\mathrm{C} 25-\mathrm{H} 25 \mathrm{C}$ & 109.5 \\
\hline $\mathrm{C} 4-\mathrm{N} 4-\mathrm{N} 3$ & $108.2(4)$ & $\mathrm{H} 25 \mathrm{~A}-\mathrm{C} 25-\mathrm{H} 25 \mathrm{C}$ & 109.5 \\
\hline $\mathrm{C} 4-\mathrm{N} 4-\mathrm{Cu} 3$ & $129.9(4)$ & $\mathrm{H} 25 \mathrm{~B}-\mathrm{C} 25-\mathrm{H} 25 \mathrm{C}$ & 109.5 \\
\hline $\mathrm{N} 3-\mathrm{N} 4-\mathrm{Cu} 3$ & $121.9(3)$ & $\mathrm{N} 8-\mathrm{C} 26-\mathrm{C} 27$ & $115.2(6)$ \\
\hline $\mathrm{C} 9-\mathrm{N} 5-\mathrm{N} 6$ & $108.6(4)$ & $\mathrm{N} 8-\mathrm{C} 26-\mathrm{H} 26 \mathrm{~A}$ & 108.5 \\
\hline $\mathrm{C} 9-\mathrm{N} 5-\mathrm{Cu} 3$ & $131.5(4)$ & $\mathrm{C} 27-\mathrm{C} 26-\mathrm{H} 26 \mathrm{~A}$ & 108.5 \\
\hline $\mathrm{N} 6-\mathrm{N} 5-\mathrm{Cu} 3$ & $119.9(3)$ & $\mathrm{N} 8-\mathrm{C} 26-\mathrm{H} 26 \mathrm{~B}$ & 108.5 \\
\hline $\mathrm{C} 7-\mathrm{N} 6-\mathrm{N} 5$ & $108.1(5)$ & $\mathrm{C} 27-\mathrm{C} 26-\mathrm{H} 26 \mathrm{~B}$ & 108.5 \\
\hline $\mathrm{C} 7-\mathrm{N} 6-\mathrm{Cu} 1$ & $128.9(4)$ & $\mathrm{H} 26 \mathrm{~A}-\mathrm{C} 26-\mathrm{H} 26 \mathrm{~B}$ & 107.5 \\
\hline $\mathrm{N} 5-\mathrm{N} 6-\mathrm{Cu} 1$ & $122.9(4)$ & $\mathrm{C} 26-\mathrm{C} 27-\mathrm{C} 28 \mathrm{~A}$ & $119.7(14)$ \\
\hline $\mathrm{C} 22-\mathrm{N} 7-\mathrm{C} 18$ & $110.2(5)$ & $\mathrm{C} 26-\mathrm{C} 27-\mathrm{C} 28 \mathrm{~B}$ & $102.4(11)$ \\
\hline $\mathrm{C} 22-\mathrm{N} 7-\mathrm{C} 10$ & $107.2(5)$ & $\mathrm{C} 26-\mathrm{C} 27-\mathrm{H} 27 \mathrm{~A}$ & 107.4 \\
\hline $\mathrm{C} 18-\mathrm{N} 7-\mathrm{C} 10$ & $111.3(6)$ & $\mathrm{C} 28 \mathrm{~A}-\mathrm{C} 27-\mathrm{H} 27 \mathrm{~A}$ & 107.4 \\
\hline $\mathrm{C} 22-\mathrm{N} 7-\mathrm{C} 14$ & $110.6(5)$ & $\mathrm{C} 26-\mathrm{C} 27-\mathrm{H} 27 \mathrm{~B}$ & 107.4 \\
\hline $\mathrm{C} 18-\mathrm{N} 7-\mathrm{C} 14$ & $106.9(5)$ & $\mathrm{C} 28 \mathrm{~A}-\mathrm{C} 27-\mathrm{H} 27 \mathrm{~B}$ & 107.4 \\
\hline $\mathrm{C} 10-\mathrm{N} 7-\mathrm{C} 14$ & $110.7(5)$ & $\mathrm{H} 27 \mathrm{~A}-\mathrm{C} 27-\mathrm{H} 27 \mathrm{~B}$ & 106.9 \\
\hline $\mathrm{C} 34-\mathrm{N} 8-\mathrm{C} 26$ & $110.9(5)$ & $\mathrm{C} 26-\mathrm{C} 27-\mathrm{H} 27 \mathrm{C}$ & 111.3 \\
\hline $\mathrm{C} 34-\mathrm{N} 8-\mathrm{C} 38$ & $110.5(5)$ & $\mathrm{C} 28 \mathrm{~B}-\mathrm{C} 27-\mathrm{H} 27 \mathrm{C}$ & 111.3 \\
\hline $\mathrm{C} 26-\mathrm{N} 8-\mathrm{C} 38$ & $107.2(5)$ & $\mathrm{C} 26-\mathrm{C} 27-\mathrm{H} 27 \mathrm{D}$ & 111.3 \\
\hline $\mathrm{C} 34-\mathrm{N} 8-\mathrm{C} 30$ & $105.6(5)$ & $\mathrm{C} 28 \mathrm{~B}-\mathrm{C} 27-\mathrm{H} 27 \mathrm{D}$ & 111.3 \\
\hline $\mathrm{C} 26-\mathrm{N} 8-\mathrm{C} 30$ & $111.2(5)$ & $\mathrm{H} 27 \mathrm{C}-\mathrm{C} 27-\mathrm{H} 27 \mathrm{D}$ & 109.2 \\
\hline $\mathrm{C} 38-\mathrm{N} 8-\mathrm{C} 30$ & $111.5(5)$ & $\mathrm{C} 29 \mathrm{~A}-\mathrm{C} 28 \mathrm{~A}-\mathrm{C} 27$ & $100.9(15)$ \\
\hline $\mathrm{N} 2-\mathrm{C} 1-\mathrm{C} 2$ & $108.6(5)$ & $\mathrm{C} 29 \mathrm{~A}-\mathrm{C} 28 \mathrm{~A}-\mathrm{H} 28 \mathrm{~A}$ & 111.6 \\
\hline $\mathrm{N} 2-\mathrm{C} 1-\mathrm{H} 1$ & 125.7 & $\mathrm{C} 27-\mathrm{C} 28 \mathrm{~A}-\mathrm{H} 28 \mathrm{~A}$ & 111.6 \\
\hline $\mathrm{C} 2-\mathrm{C} 1-\mathrm{H} 1$ & 125.7 & $\mathrm{C} 29 \mathrm{~A}-\mathrm{C} 28 \mathrm{~A}-\mathrm{H} 28 \mathrm{~B}$ & 111.6 \\
\hline $\mathrm{C} 3-\mathrm{C} 2-\mathrm{C} 1$ & $105.2(5)$ & $\mathrm{C} 27-\mathrm{C} 28 \mathrm{~A}-\mathrm{H} 28 \mathrm{~B}$ & 111.6 \\
\hline $\mathrm{C} 3-\mathrm{C} 2-\mathrm{I} 1$ & $124.5(4)$ & $\mathrm{H} 28 \mathrm{~A}-\mathrm{C} 28 \mathrm{~A}-\mathrm{H} 28 \mathrm{~B}$ & 109.4 \\
\hline $\mathrm{C} 1-\mathrm{C} 2-\mathrm{I} 1$ & $129.8(4)$ & $\mathrm{C} 28 \mathrm{~A}-\mathrm{C} 29 \mathrm{~A}-\mathrm{H} 29 \mathrm{~A}$ & 109.5 \\
\hline $\mathrm{N} 1-\mathrm{C} 3-\mathrm{C} 2$ & $109.2(5)$ & $\mathrm{C} 28 \mathrm{~A}-\mathrm{C} 29 \mathrm{~A}-\mathrm{H} 29 \mathrm{~B}$ & 109.5 \\
\hline $\mathrm{N} 1-\mathrm{C} 3-\mathrm{H} 3$ & 125.4 & $\mathrm{H} 29 \mathrm{~A}-\mathrm{C} 29 \mathrm{~A}-\mathrm{H} 29 \mathrm{~B}$ & 109.5 \\
\hline $\mathrm{C} 2-\mathrm{C} 3-\mathrm{H} 3$ & 125.4 & $\mathrm{C} 28 \mathrm{~A}-\mathrm{C} 29 \mathrm{~A}-\mathrm{H} 29 \mathrm{C}$ & 109.5 \\
\hline $\mathrm{N} 4-\mathrm{C} 4-\mathrm{C} 5$ & $109.2(5)$ & $\mathrm{H} 29 \mathrm{~A}-\mathrm{C} 29 \mathrm{~A}-\mathrm{H} 29 \mathrm{C}$ & 109.5 \\
\hline $\mathrm{N} 4-\mathrm{C} 4-\mathrm{H} 4$ & 125.4 & $\mathrm{H} 29 \mathrm{~B}-\mathrm{C} 29 \mathrm{~A}-\mathrm{H} 29 \mathrm{C}$ & 109.5 \\
\hline
\end{tabular}




\begin{tabular}{|c|c|}
\hline $\mathrm{C} 5-\mathrm{C} 4-\mathrm{H} 4$ & 125.4 \\
\hline $\mathrm{C} 6-\mathrm{C} 5-\mathrm{C} 4$ & $105.0(5)$ \\
\hline $\mathrm{C} 6-\mathrm{C} 5-\mathrm{I} 2$ & $127.8(4)$ \\
\hline $\mathrm{C} 4-\mathrm{C} 5-\mathrm{I} 2$ & $127.0(4)$ \\
\hline $\mathrm{N} 3-\mathrm{C} 6-\mathrm{C} 5$ & $109.6(5)$ \\
\hline $\mathrm{N} 3-\mathrm{C} 6-\mathrm{H} 6$ & 125.2 \\
\hline $\mathrm{C} 5-\mathrm{C} 6-\mathrm{H} 6$ & 125.2 \\
\hline $\mathrm{N} 6-\mathrm{C} 7-\mathrm{C} 8$ & $109.2(5)$ \\
\hline $\mathrm{N} 6-\mathrm{C} 7-\mathrm{H} 7$ & 125.4 \\
\hline $\mathrm{C} 8-\mathrm{C} 7-\mathrm{H} 7$ & 125.4 \\
\hline $\mathrm{C} 7-\mathrm{C} 8-\mathrm{C} 9$ & $105.0(5)$ \\
\hline $\mathrm{C} 7-\mathrm{C} 8-\mathrm{I} 3$ & $124.3(4)$ \\
\hline $\mathrm{C} 9-\mathrm{C} 8-\mathrm{I} 3$ & $130.7(4)$ \\
\hline $\mathrm{N} 5-\mathrm{C} 9-\mathrm{C} 8$ & $109.1(5)$ \\
\hline $\mathrm{N} 5-\mathrm{C} 9-\mathrm{H} 9$ & 125.5 \\
\hline $\mathrm{C} 8-\mathrm{C} 9-\mathrm{H} 9$ & 125.5 \\
\hline $\mathrm{C} 11-\mathrm{C} 10-\mathrm{N} 7$ & $115.4(7)$ \\
\hline $\mathrm{C} 11-\mathrm{C} 10-\mathrm{H} 10 \mathrm{~A}$ & 108.4 \\
\hline $\mathrm{N} 7-\mathrm{C} 10-\mathrm{H} 10 \mathrm{~A}$ & 108.4 \\
\hline $\mathrm{C} 11-\mathrm{C} 10-\mathrm{H} 10 \mathrm{~B}$ & 108.4 \\
\hline N7-C10-H10B & 108.4 \\
\hline $\mathrm{H} 10 \mathrm{~A}-\mathrm{C} 10-\mathrm{H} 10 \mathrm{~B}$ & 107.5 \\
\hline $\mathrm{C} 10-\mathrm{C} 11-\mathrm{C} 12 \mathrm{~B}$ & $132.7(18)$ \\
\hline $\mathrm{C} 10-\mathrm{C} 11-\mathrm{C} 12 \mathrm{~A}$ & $103.6(10)$ \\
\hline $\mathrm{C} 10-\mathrm{C} 11-\mathrm{H} 11 \mathrm{~A}$ & 111.0 \\
\hline $\mathrm{C} 12 \mathrm{~A}-\mathrm{C} 11-\mathrm{H} 11 \mathrm{~A}$ & 111.0 \\
\hline $\mathrm{C} 10-\mathrm{C} 11-\mathrm{H} 11 \mathrm{~B}$ & 111.0 \\
\hline $\mathrm{C} 12 \mathrm{~A}-\mathrm{C} 11-\mathrm{H} 11 \mathrm{~B}$ & 111.0 \\
\hline $\mathrm{H} 11 \mathrm{~A}-\mathrm{C} 11-\mathrm{H} 11 \mathrm{~B}$ & 109.0 \\
\hline $\mathrm{C} 10-\mathrm{C} 11-\mathrm{H} 11 \mathrm{C}$ & 104.1 \\
\hline $\mathrm{C} 12 \mathrm{~B}-\mathrm{C} 11-\mathrm{H} 11 \mathrm{C}$ & 104.1 \\
\hline $\mathrm{C} 10-\mathrm{C} 11-\mathrm{H} 11 \mathrm{D}$ & 104.1 \\
\hline $\mathrm{C} 12 \mathrm{~B}-\mathrm{C} 11-\mathrm{H} 11 \mathrm{D}$ & 104.1 \\
\hline $\mathrm{H} 11 \mathrm{C}-\mathrm{C} 11-\mathrm{H} 11 \mathrm{D}$ & 105.5 \\
\hline $\mathrm{C} 11-\mathrm{C} 12 \mathrm{~A}-\mathrm{C} 13 \mathrm{~A}$ & $103.6(12)$ \\
\hline $\mathrm{C} 11-\mathrm{C} 12 \mathrm{~A}-\mathrm{H} 12 \mathrm{~A}$ & 111.1 \\
\hline $\mathrm{C} 13 \mathrm{~A}-\mathrm{C} 12 \mathrm{~A}-\mathrm{H} 12 \mathrm{~A}$ & 111.1 \\
\hline $\mathrm{C} 11-\mathrm{C} 12 \mathrm{~A}-\mathrm{H} 12 \mathrm{~B}$ & 111.0 \\
\hline $\mathrm{C} 13 \mathrm{~A}-\mathrm{C} 12 \mathrm{~A}-\mathrm{H} 12 \mathrm{~B}$ & 111.0 \\
\hline $\mathrm{H} 12 \mathrm{~A}-\mathrm{C} 12 \mathrm{~A}-\mathrm{H} 12 \mathrm{~B}$ & 109.0 \\
\hline $\mathrm{C} 12 \mathrm{~A}-\mathrm{C} 13 \mathrm{~A}-\mathrm{H} 13 \mathrm{~A}$ & 109.5 \\
\hline $\mathrm{C} 12 \mathrm{~A}-\mathrm{C} 13 \mathrm{~A}-\mathrm{H} 13 \mathrm{~B}$ & 109.5 \\
\hline $\mathrm{H} 13 \mathrm{~A}-\mathrm{C} 13 \mathrm{~A}-\mathrm{H} 13 \mathrm{~B}$ & 109.5 \\
\hline $\mathrm{C} 12 \mathrm{~A}-\mathrm{C} 13 \mathrm{~A}-\mathrm{H} 13 \mathrm{C}$ & 109.5 \\
\hline $\mathrm{H} 13 \mathrm{~A}-\mathrm{C} 13 \mathrm{~A}-\mathrm{H} 13 \mathrm{C}$ & 109.5 \\
\hline $\mathrm{H} 13 \mathrm{~B}-\mathrm{C} 13 \mathrm{~A}-\mathrm{H} 13 \mathrm{C}$ & 109.5 \\
\hline $\mathrm{C} 11-\mathrm{C} 12 \mathrm{~B}-\mathrm{C} 13 \mathrm{~B}$ & $102.0(19)$ \\
\hline & \\
\hline
\end{tabular}

$\mathrm{C} 5-\mathrm{C} 4-\mathrm{H} 4$

C6- $5-\mathrm{C} 4$

$\mathrm{N} 3-\mathrm{C} 6-\mathrm{C} 5$

$\mathrm{N} 3-\mathrm{C} 6-\mathrm{H} 6$

$\mathrm{C} 5-\mathrm{C} 6-\mathrm{H} 6$

$\mathrm{N} 6-\mathrm{C} 7-\mathrm{C} 8$

$\mathrm{N} 6-\mathrm{C} 7-\mathrm{H} 7$

$\mathrm{C} 7-\mathrm{C} 8-\mathrm{C} 9$

$\mathrm{C} 7-\mathrm{C} 8-\mathrm{I} 3$

$\mathrm{N} 5-\mathrm{C} 9-\mathrm{H} 9$

$\mathrm{C} 8-\mathrm{C} 9-\mathrm{H} 9$

$\mathrm{C} 11-\mathrm{C} 10-\mathrm{N} 7$

$\mathrm{C} 11-\mathrm{C} 10-\mathrm{H} 10 \mathrm{~A}$

$\mathrm{C} 11-\mathrm{C} 10-\mathrm{H} 10 \mathrm{~B}$

N7- $\mathrm{C} 10-\mathrm{H} 10 \mathrm{~B}$

$\mathrm{C} 10-\mathrm{C} 11-\mathrm{C} 12 \mathrm{~B}$

$\mathrm{C} 12 \mathrm{~A}-\mathrm{C} 11-\mathrm{H} 11 \mathrm{~A}$

$\mathrm{C} 10-\mathrm{C} 11-\mathrm{H} 11 \mathrm{~B}$

$\mathrm{C} 12 \mathrm{~A}-\mathrm{C} 11-\mathrm{H} 11 \mathrm{~B}$

$\mathrm{C} 12 \mathrm{~B}-\mathrm{C} 11-\mathrm{H} 11 \mathrm{C}$

$\mathrm{H} 11 \mathrm{C}-\mathrm{C} 11-\mathrm{H} 11 \mathrm{D}$

C11-C12A-C13A
125.4

$127.8(4)$

$127.0(4)$

$109.6(5)$

125.2

125.2

$109.2(5)$

125.4

$105.0(5)$

(4)

$109.1(5)$

25.5

25.5

15.4 (7)

108.4

108.4

108.4

108.4

132.7 (18)

$103.6(10)$

111.0

111.0

111.0

109.0

104.1

104.1

104.1

104.1

105.5

$103.6(12)$

111.1

111.0

109.0

109.5

109.5

109.5

109.5

109.5

102.0 (19)

111.4

\begin{tabular}{|c|c|}
\hline $\mathrm{C} 29 \mathrm{~B}-\mathrm{C} 28 \mathrm{~B}-\mathrm{C} 27$ & $106.9(15)$ \\
\hline $\mathrm{C} 29 \mathrm{~B}-\mathrm{C} 28 \mathrm{~B}-\mathrm{H} 28 \mathrm{C}$ & 110.3 \\
\hline $\mathrm{C} 27-\mathrm{C} 28 \mathrm{~B}-\mathrm{H} 28 \mathrm{C}$ & 110.4 \\
\hline $\mathrm{C} 29 \mathrm{~B}-\mathrm{C} 28 \mathrm{~B}-\mathrm{H} 28 \mathrm{D}$ & 110.4 \\
\hline $\mathrm{C} 27-\mathrm{C} 28 \mathrm{~B}-\mathrm{H} 28 \mathrm{D}$ & 110.4 \\
\hline $\mathrm{H} 28 \mathrm{C}-\mathrm{C} 28 \mathrm{~B}-\mathrm{H} 28 \mathrm{D}$ & 108.6 \\
\hline $\mathrm{C} 28 \mathrm{~B}-\mathrm{C} 29 \mathrm{~B}-\mathrm{H} 29 \mathrm{D}$ & 109.5 \\
\hline $\mathrm{C} 28 \mathrm{~B}-\mathrm{C} 29 \mathrm{~B}-\mathrm{H} 29 \mathrm{E}$ & 109.5 \\
\hline $\mathrm{H} 29 \mathrm{D}-\mathrm{C} 29 \mathrm{~B}-\mathrm{H} 29 \mathrm{E}$ & 109.5 \\
\hline $\mathrm{C} 28 \mathrm{~B}-\mathrm{C} 29 \mathrm{~B}-\mathrm{H} 29 \mathrm{~F}$ & 109.5 \\
\hline $\mathrm{H} 29 \mathrm{D}-\mathrm{C} 29 \mathrm{~B}-\mathrm{H} 29 \mathrm{~F}$ & 109.5 \\
\hline $\mathrm{H} 29 \mathrm{E}-\mathrm{C} 29 \mathrm{~B}-\mathrm{H} 29 \mathrm{~F}$ & 109.5 \\
\hline $\mathrm{C} 31-\mathrm{C} 30-\mathrm{N} 8$ & $115.1(6)$ \\
\hline $\mathrm{C} 31-\mathrm{C} 30-\mathrm{H} 30 \mathrm{~A}$ & 108.5 \\
\hline $\mathrm{N} 8-\mathrm{C} 30-\mathrm{H} 30 \mathrm{~A}$ & 108.5 \\
\hline $\mathrm{C} 31-\mathrm{C} 30-\mathrm{H} 30 \mathrm{~B}$ & 108.5 \\
\hline $\mathrm{N} 8-\mathrm{C} 30-\mathrm{H} 30 \mathrm{~B}$ & 108.5 \\
\hline $\mathrm{H} 30 \mathrm{~A}-\mathrm{C} 30-\mathrm{H} 30 \mathrm{~B}$ & 107.5 \\
\hline $\mathrm{C} 30-\mathrm{C} 31-\mathrm{C} 32$ & $110.0(7)$ \\
\hline $\mathrm{C} 30-\mathrm{C} 31-\mathrm{H} 31 \mathrm{~A}$ & 109.7 \\
\hline $\mathrm{C} 32-\mathrm{C} 31-\mathrm{H} 31 \mathrm{~A}$ & 109.7 \\
\hline $\mathrm{C} 30-\mathrm{C} 31-\mathrm{H} 31 \mathrm{~B}$ & 109.7 \\
\hline $\mathrm{C} 32-\mathrm{C} 31-\mathrm{H} 31 \mathrm{~B}$ & 109.7 \\
\hline $\mathrm{H} 31 \mathrm{~A}-\mathrm{C} 31-\mathrm{H} 31 \mathrm{~B}$ & 108.2 \\
\hline $\mathrm{C} 33-\mathrm{C} 32-\mathrm{C} 31$ & $114.8(10)$ \\
\hline $\mathrm{C} 33-\mathrm{C} 32-\mathrm{H} 32 \mathrm{~A}$ & 108.6 \\
\hline $\mathrm{C} 31-\mathrm{C} 32-\mathrm{H} 32 \mathrm{~A}$ & 108.6 \\
\hline C $33-\mathrm{C} 32-\mathrm{H} 32 \mathrm{~B}$ & 108.6 \\
\hline $\mathrm{C} 31-\mathrm{C} 32-\mathrm{H} 32 \mathrm{~B}$ & 108.6 \\
\hline $\mathrm{H} 32 \mathrm{~A}-\mathrm{C} 32-\mathrm{H} 32 \mathrm{~B}$ & 107.6 \\
\hline $\mathrm{C} 32-\mathrm{C} 33-\mathrm{H} 33 \mathrm{~A}$ & 109.5 \\
\hline $\mathrm{C} 32-\mathrm{C} 33-\mathrm{H} 33 \mathrm{~B}$ & 109.5 \\
\hline $\mathrm{H} 33 \mathrm{~A}-\mathrm{C} 33-\mathrm{H} 33 \mathrm{~B}$ & 109.5 \\
\hline $\mathrm{C} 32-\mathrm{C} 33-\mathrm{H} 33 \mathrm{C}$ & 109.5 \\
\hline $\mathrm{H} 33 \mathrm{~A}-\mathrm{C} 33-\mathrm{H} 33 \mathrm{C}$ & 109.5 \\
\hline $\mathrm{H} 33 \mathrm{~B}-\mathrm{C} 33-\mathrm{H} 33 \mathrm{C}$ & 109.5 \\
\hline $\mathrm{N} 8-\mathrm{C} 34-\mathrm{C} 35$ & $116.1(5)$ \\
\hline $\mathrm{N} 8-\mathrm{C} 34-\mathrm{H} 34 \mathrm{~A}$ & 108.3 \\
\hline $\mathrm{C} 35-\mathrm{C} 34-\mathrm{H} 34 \mathrm{~A}$ & 108.3 \\
\hline $\mathrm{N} 8-\mathrm{C} 34-\mathrm{H} 34 \mathrm{~B}$ & 108.3 \\
\hline $\mathrm{C} 35-\mathrm{C} 34-\mathrm{H} 34 \mathrm{~B}$ & 108.3 \\
\hline $\mathrm{H} 34 \mathrm{~A}-\mathrm{C} 34-\mathrm{H} 34 \mathrm{~B}$ & 107.4 \\
\hline $\mathrm{C} 36-\mathrm{C} 35-\mathrm{C} 34$ & $110.5(6)$ \\
\hline $\mathrm{C} 36-\mathrm{C} 35-\mathrm{H} 35 \mathrm{~A}$ & 109.5 \\
\hline $\mathrm{C} 34-\mathrm{C} 35-\mathrm{H} 35 \mathrm{~A}$ & 109.5 \\
\hline $\mathrm{C} 36-\mathrm{C} 35-\mathrm{H} 35 \mathrm{~B}$ & 109.5 \\
\hline $\mathrm{C} 34-\mathrm{C} 35-\mathrm{H} 35 \mathrm{~B}$ & 109.5 \\
\hline $\mathrm{H} 35 \mathrm{~A}-\mathrm{C} 35-\mathrm{H} 35 \mathrm{~B}$ & 108.1 \\
\hline
\end{tabular}


$\mathrm{C} 13 \mathrm{~B}-\mathrm{C} 12 \mathrm{~B}-\mathrm{H} 12 \mathrm{C}$

C11-C12B-H12D

$\mathrm{C} 13 \mathrm{~B}-\mathrm{C} 12 \mathrm{~B}-\mathrm{H} 12 \mathrm{D}$

$\mathrm{H} 12 \mathrm{C}-\mathrm{C} 12 \mathrm{~B}-\mathrm{H} 12 \mathrm{D}$

C12B-C13B-H13D

$\mathrm{C} 12 \mathrm{~B}-\mathrm{C} 13 \mathrm{~B}-\mathrm{H} 13 \mathrm{E}$

H13D-C13B-H13E

$\mathrm{C} 12 \mathrm{~B}-\mathrm{C} 13 \mathrm{~B}-\mathrm{H} 13 \mathrm{~F}$

$\mathrm{H} 13 \mathrm{D}-\mathrm{C} 13 \mathrm{~B}-\mathrm{H} 13 \mathrm{~F}$

$\mathrm{H} 13 \mathrm{E}-\mathrm{C} 13 \mathrm{~B}-\mathrm{H} 13 \mathrm{~F}$

N7-C14-C15

N7-C14-H14A

C15-C14-H14A

N7-C14-H14B

C15-C14-H14B

$\mathrm{H} 14 \mathrm{~A}-\mathrm{C} 14-\mathrm{H} 14 \mathrm{~B}$

$\mathrm{C} 14-\mathrm{C} 15-\mathrm{C} 16 \mathrm{~A}$

$\mathrm{C} 14-\mathrm{C} 15-\mathrm{C} 16 \mathrm{~B}$

C14-C15-H15A

$\mathrm{C} 16 \mathrm{~A}-\mathrm{C} 15-\mathrm{H} 15 \mathrm{~A}$

C14-C15-H15B

$\mathrm{C} 16 \mathrm{~A}-\mathrm{C} 15-\mathrm{H} 15 \mathrm{~B}$

H15A-C15-H15B

C14-C15-H15C

$\mathrm{C} 16 \mathrm{~B}-\mathrm{C} 15-\mathrm{H} 15 \mathrm{C}$

C14-C15-H15D

C16B-C15-H15D

$\mathrm{H} 15 \mathrm{C}-\mathrm{C} 15-\mathrm{H} 15 \mathrm{D}$

$\mathrm{C} 17 \mathrm{~A}-\mathrm{C} 16 \mathrm{~A}-\mathrm{C} 15$

$\mathrm{C} 17 \mathrm{~A}-\mathrm{C} 16 \mathrm{~A}-\mathrm{H} 16 \mathrm{~A}$

C15-C16A-H16A

C17A-C16A-H16B

C15-C16A-H16B

H16A-C16A-H16B

C16A-C17A-H17A

C16A-C17A-H17B

H17A-C17A-H17B

C16A-C17A-H17C

H17A-C17A-H17C

$\mathrm{H} 17 \mathrm{~B}-\mathrm{C} 17 \mathrm{~A}-\mathrm{H} 17 \mathrm{C}$

$\mathrm{C} 17 \mathrm{~B}-\mathrm{C} 16 \mathrm{~B}-\mathrm{C} 15$

$\mathrm{C} 17 \mathrm{~B}-\mathrm{C} 16 \mathrm{~B}-\mathrm{H} 16 \mathrm{C}$

C15-C16B-H16C

$\mathrm{C} 17 \mathrm{~B}-\mathrm{C} 16 \mathrm{~B}-\mathrm{H} 16 \mathrm{D}$

C15-C16B-H16D

$\mathrm{H} 16 \mathrm{C}-\mathrm{C} 16 \mathrm{~B}-\mathrm{H} 16 \mathrm{D}$
111.4

111.4

111.4

109.2

109.5

109.5

109.5

109.5

109.5

109.5

116.5 (6)

108.2

108.2

108.2

108.2

107.3

108.5 (11)

109.2 (15)

110.0

110.0

110.0

110.0

108.4

109.8

109.8

109.8

109.8

108.3

115.9 (18)

108.3

108.3

108.3

108.3

107.4

109.5

109.5

109.5

109.5

109.5

109.5

106.0 (19)

110.5

110.5

110.5

110.5

108.7
C35-C36-C37

C $35-\mathrm{C} 36-\mathrm{H} 36 \mathrm{~A}$

$\mathrm{C} 37-\mathrm{C} 36-\mathrm{H} 36 \mathrm{~A}$

C $35-\mathrm{C} 36-\mathrm{H} 36 \mathrm{~B}$

C $37-\mathrm{C} 36-\mathrm{H} 36 \mathrm{~B}$

$\mathrm{H} 36 \mathrm{~A}-\mathrm{C} 36-\mathrm{H} 36 \mathrm{~B}$

C36-C $37-\mathrm{H} 37 \mathrm{~A}$

C36-C37- H37B

H37A-C37-H37B

C36-C37- $337 \mathrm{C}$

H37A-C37- H37C

H37B-C $37-\mathrm{H} 37 \mathrm{C}$

N8-C38-C39

N8-C38-H38A

C39-C $38-\mathrm{H} 38 \mathrm{~A}$

$\mathrm{N} 8-\mathrm{C} 38-\mathrm{H} 38 \mathrm{~B}$

C39-C $38-\mathrm{H} 38 \mathrm{~B}$

H38A-C38-H38B

$\mathrm{C} 40-\mathrm{C} 39-\mathrm{C} 38$

C40-C39-H39A

C38-C39-H39A

C40-C39- H39B

C38-C39- H39B

H39A-C39-H39B

$\mathrm{C} 41-\mathrm{C} 40-\mathrm{C} 39$

$\mathrm{C} 41-\mathrm{C} 40-\mathrm{H} 40 \mathrm{~A}$

C39- $440-\mathrm{H} 40 \mathrm{~A}$

$\mathrm{C} 41-\mathrm{C} 40-\mathrm{H} 40 \mathrm{~B}$

C39- $440-\mathrm{H} 40 \mathrm{~B}$

$\mathrm{H} 40 \mathrm{~A}-\mathrm{C} 40-\mathrm{H} 40 \mathrm{~B}$

$\mathrm{C} 40-\mathrm{C} 41-\mathrm{H} 41 \mathrm{~A}$

C40-C41- H41B

$\mathrm{H} 41 \mathrm{~A}-\mathrm{C} 41-\mathrm{H} 41 \mathrm{~B}$

$\mathrm{C} 40-\mathrm{C} 41-\mathrm{H} 41 \mathrm{C}$

$\mathrm{H} 41 \mathrm{~A}-\mathrm{C} 41-\mathrm{H} 41 \mathrm{C}$

$\mathrm{H} 41 \mathrm{~B}-\mathrm{C} 41-\mathrm{H} 41 \mathrm{C}$

$\mathrm{O} 1-\mathrm{C} 42-\mathrm{C}_{4} 3^{\mathrm{i}}$

$\mathrm{O} 1-\mathrm{C} 42-\mathrm{H} 42 \mathrm{~A}$

$\mathrm{C} 43^{\mathrm{i}}-\mathrm{C} 42-\mathrm{H} 42 \mathrm{~A}$

$\mathrm{O} 1-\mathrm{C} 42-\mathrm{H} 42 \mathrm{~B}$

$\mathrm{C} 43^{\mathrm{i}}-\mathrm{C} 42-\mathrm{H} 42 \mathrm{~B}$

$\mathrm{H} 42 \mathrm{~A}-\mathrm{C} 42-\mathrm{H} 42 \mathrm{~B}$

$\mathrm{O} 1-\mathrm{C} 43-\mathrm{C} 42^{\mathrm{i}}$

$\mathrm{O} 1-\mathrm{C} 43-\mathrm{H} 43 \mathrm{~A}$

$\mathrm{C} 42^{\mathrm{i}}-\mathrm{C} 43-\mathrm{H} 43 \mathrm{~A}$

$\mathrm{O} 1-\mathrm{C} 43-\mathrm{H} 43 \mathrm{~B}$
113.0 (6)

109.0

109.0

109.0

109.0

107.8

109.5

109.5

109.5

109.5

109.5

109.5

115.2 (5)

108.5

108.5

108.5

108.5

107.5

$111.0(7)$

109.4

109.4

109.4

109.4

108.0

116.4 (10)

108.2

108.2

108.2

108.2

107.3

109.5

109.5

109.5

109.5

109.5

109.5

110.4 (6)

109.6

109.6

109.6

109.6

108.1

110.5 (6)

109.6

109.6

109.6 
C16B-C17B-H17D

C16B-C17B-H17E

Symmetry code: (i) $-x+2,-y,-z+1$.
109.5

109.5
$\mathrm{C} 42^{\mathrm{i}}-\mathrm{C} 43-\mathrm{H} 43 \mathrm{~B}$

$\mathrm{H} 43 \mathrm{~A}-\mathrm{C} 43-\mathrm{H} 43 \mathrm{~B}$
109.6

108.1 